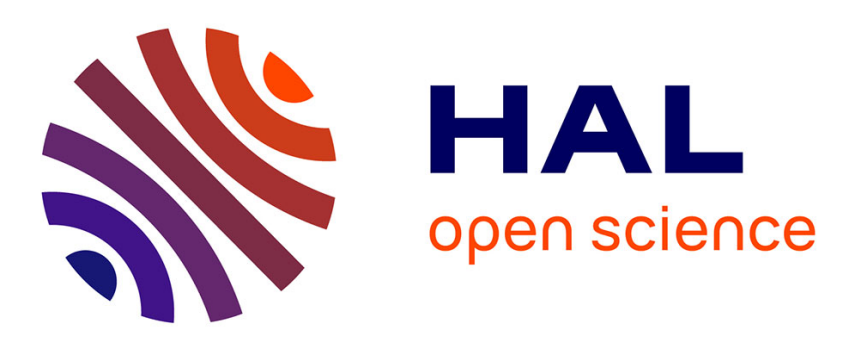

\title{
Numerical analysis of strain localization in Rocks with Thermo-Hydro-Mechanical couplings using Cosserat continuum.
}

Hadrien Rattez, Ioannis Stefanou, Jean Sulem, Manolis Veveakis, Thomas Poulet

\section{To cite this version:}

Hadrien Rattez, Ioannis Stefanou, Jean Sulem, Manolis Veveakis, Thomas Poulet. Numerical analysis of strain localization in Rocks with Thermo-Hydro-Mechanical couplings using Cosserat continuum.. Rock Mechanics and Rock Engineering, 2018, 10.1007/s00603-018-1529-7 . hal-01821909

\section{HAL Id: hal-01821909 \\ https://hal-enpc.archives-ouvertes.fr/hal-01821909}

Submitted on 23 Jun 2018

HAL is a multi-disciplinary open access archive for the deposit and dissemination of scientific research documents, whether they are published or not. The documents may come from teaching and research institutions in France or abroad, or from public or private research centers.
L'archive ouverte pluridisciplinaire HAL, est destinée au dépôt et à la diffusion de documents scientifiques de niveau recherche, publiés ou non, émanant des établissements d'enseignement et de recherche français ou étrangers, des laboratoires publics ou privés. 
See discussions, stats, and author profiles for this publication at: https://www.researchgate.net/publication/325905084

\section{Numerical analysis of strain localization in Rocks with Thermo-Hydro- Mechanical couplings using Cosserat continuum}

Article in Rock Mechanics and Rock Engineering · June 2018

CITATIONS

0

5 authors, including:

Hadrien Rattez

Duke University

13 PUBLICATIONS 8 CITATIONS

SEE PROFILE

\section{READS}

4

6

Ioannis Stefanou

Navier Laboratory, Ecole Nationale des Ponts et Chaussées, IFSTT..

54 PUBLICATIONS 375 CITATIONS

SEE PROFILE

Some of the authors of this publication are also working on these related projects:

Thermal expansion of adsorbing microporous media and application to clay desiccation View project

GeoProc2017 View project 


\title{
Numerical analysis of strain localization in Rocks with Thermo-Hydro-Mechanical couplings using Cosserat continuum.
}

\author{
Hadrien Rattez • Ioannis Stefanou • Jean \\ Sulem - Manolis Veveakis . Thomas Poulet
}

Received: 2018

\begin{abstract}
A numerical model for Thermo-Hydro-Mechanical (THM) strong couplings in an elasto-plastic Cosserat continuum is developed in order to explore the influence of frictional heating and thermal pore fluid pressurization on the strain localization phenomenon. This model allows specifically to study the complete stress-strain response of a rock specimen as well as the size of the strain localization zone for a rock taking into account its microstructure. The numerical implementation in a finite element code is presented, matching adequately analytical solutions or results from other simulations found in the literature. Two different applications of the numerical model are also presented to highlight its capabilities. The first one is a biaxial test on a saturated weak sandstone, for which the influence on the stress-strain response of the characteristic size of the microstructure and of thermal pressurization is investigated. The second one is the rapid shearing of a mature fault zone in the brittle part of the lithosphere. In this example, the evolution of the thickness of the localized zone and the influence of the permeability change on the stress-strain response are studied.
\end{abstract}

Keywords Thermo-Hydro-Mechanical couplings · Cosserat continuum · strain localization · Finite Element Model

H. Rattez, I. Stefanou, J.Sulem Laboratoire Navier/CERMES, UMR 8205, École des Ponts ParisTech, IFSTTAR, CNRS 6/8 avenue Blaise Pascal, 77455 Marne-la-Valle, France

Tel.: +33164153456

E-mail: hadrien.rattez@enpc.fr

T. Poulet

CSIRO Mineral Resources

Kensington, WA 6151, Australia

M. Veveakis, T. Poulet

School of Petroleum Engineering, University of New South Wales, Sydney, Australia 


\section{List of Symbols}

c Cohesion

C Heat capacity of the porous material

$c_{h y} \quad$ Hydraulic diffusivity

$C_{i j k l}^{e} \quad$ Elastic stiffness tensor

$c_{h y} \quad$ Thermal diffusivity

$e_{i j k} \quad$ Levi-Civita symbol

$F \quad$ Yield surface

$G \quad$ Shear modulus

$G_{c} \quad$ Cosserat shear modulus

$g_{i} \quad$ Coefficients in the generalized plastic strain invariant

$h_{i} \quad$ Coefficients in the generalized stress invariant

$K \quad$ Bulk modulus

$L \quad$ First modulus of the elastic flexural bending rigidity tensor

$M \quad$ Second modulus of the elastic flexural bending rigidity tensor

$M_{c} \quad$ Third modulus of the elastic flexural bending rigidity tensor

$M_{i j k l}^{e} \quad$ Elastic flexural bending rigidity tensor

$n \quad$ Eulerian porosity

$p \quad$ Pore pressure

$Q \quad$ Plastic potential

$q \quad$ Hardening variable

$R \quad$ Cosserat internal length

$S \quad$ External surface of the specimen

$T \quad$ Temperature

$u_{i} \quad$ Displacements

$\alpha \quad$ Coefficient of thermal expansion of the porous medium

$\beta \quad$ Dilatancy

$\beta^{f} \quad$ Compressibility of the fluid phase per unit volume

$\beta^{s} \quad$ Compressibility of the solid phase per unit volume

$\beta^{*} \quad$ Mixture compressibility per unit volume

$\chi \quad$ Permeability of the porous medium

$\delta_{i j} \quad$ Kronecker symbol

$\eta^{f} \quad$ Viscosity of the liquid phase

$\gamma_{i j} \quad$ Strain tensor

$\gamma_{[i j]} \quad$ Antisymmetric part of the strain tensor

$\gamma_{i j}^{e} \quad$ Elastic strain tensor

$\gamma^{p} \quad$ Generalized plastic strain invariant

$\gamma_{i j}^{p} \quad$ Plastic strain tensor

$\kappa_{i j} \quad$ Curvature tensor

$\kappa_{[i j]} \quad$ Antisymmetric part of the curvature tensor

$\kappa_{i j}^{e} \quad$ Elastic part of the curvature tensor

$\kappa_{i j}^{p} \quad$ Plastic part of the curvature tensor

$\kappa_{(i j)} \quad$ Symmetric part of the curvature tensor

$\lambda^{f} \quad$ Coefficient of thermal expansion of the fluid phase per unit volume

$\lambda^{s} \quad$ Coefficient of thermal expansion of the solid phase per unit volume

$\lambda^{*} \quad$ Coefficient of thermal expansion of the soil-water mixture per unit volume

$\mu \quad$ Friction coefficient

$\mu_{i j} \quad$ Couple-stress tensor 
$\Omega \quad$ Volume of the specimen

$\omega_{i}^{c} \quad$ Cosserat rotations

$\omega_{i j}^{c} \quad$ Tensor of rotations of the microstructure

$\Omega_{i j} \quad$ Macroscopic rotation tensor

$\psi_{i} \quad$ Test functions

$\rho \quad$ Density of the porous material

$\sigma^{\prime} \quad$ Effective mean stress

$\sigma_{i j} \quad$ Symmetric part of the total stress tensor

$\tau_{i j} \quad$ Total stress tensor

$\tau_{[i j]} \quad$ Antisymmetric part of the total stress tensor

$\tau \quad$ Generalized deviatoric stress invariant for Cosserat continua

$\tau_{i j}^{\prime} \quad$ Effective stress tensor

$\varepsilon_{i j} \quad$ Symmetric part of the strain tensor

$\xi \quad$ Parameter that enables to switch between different hardening

\section{Introduction}

Thermo-Hydro-Mechanical (THM) couplings play a major role in various applications in rock mechanics like for instance reservoir mechanics (Longuemare et al, 2002), fault mechanics (Sulem et al, 2011), geothermal energy (Tung et al, 2017) or

5 nuclear waste disposals (Menaceur et al, 2016). The reason is that the conditions of temperature and the presence of pore fluids greatly affect the mechanical response and vice versa. These couplings are particularly relevant in the context of strain localization, which is one of the most important feature encountered in the rocks of the lithosphere and is encountered at different scales (Regenauer-Lieb et al, 2017).

10 Beyond some level of deformation, the strength of the material decreases and this softening of the rock resistance leads to a localization of the deformation into narrow zones. This softening behavior of the material is not limited to mechanical processes and can be enhanced by multi-physical couplings.

A first approach to study strain localization consists in looking at the possible critical conditions for which the constitutive equations of the material allow the formation of deformation bands (Rice, 1975). In many cases, though, it is interesting to track the evolution of the system after the onset of localization. The full stress-strain response of the material is essential in many rock mechanics applications (Goodman, 1989), even in the absence of THM couplings. For that 20 purpose, we need to simulate numerically the solution of a nonlinear boundary value problem, which is a challenging task due to the complications that arise due to softening. Indeed, the classical continuum theory cannot be used because the governing system of equations is ill-posed (Vardoulakis, 1985) and a regularization of the problem is needed to alleviate mesh dependency (de Borst, 1991), i.e. 25 localization in only one element.

This pathology leads to nonphysical results and can be remediated by resorting to appropriate theories (Mühlhaus and Vardoulakis, 1987; Pijaudier and Bazant, 1987; Needleman, 1988). Among these theories, Cosserat continuum is particularly suitable for modeling the microstructure of granular rocks (Papanastasiou and 30 Vardoulakis, 1992; Papanastasiou and Zervos, 2016; Stefanou et al, 2017).

In this paper, we present a numerical model that can address strain localization phenomena in rocks modeled as a Cosserat continuum and considering THM cou- 
plings. Special attention is devoted to the validation of the numerical tool against analytical solutions and numerical results from the literature. Two specific exam-

35 ples of application are also developed: biaxial loading on sandstone specimens and seismic slip in fault gouges.

\section{Constitutive model formulation}

In this section a brief summary of the governing equations is given. A more detailed presentation can be found in Stefanou et al (2017); Rattez et al (2018a).

\subsection{Cosserat continuum}

As compared to the classical (Cauchy) continuum, in a 3D-Cosserat continuum each material point has three additional rotational degrees of freedom $\omega_{k}^{c}$ related to the tensor of rotation of the microstructure $\omega_{i j}^{c}$ by:

$$
\omega_{i j}^{c}=-e_{i j k} \omega_{k}^{c}
$$

where $e_{i j k}$ is the Levi-Civita symbol and the indices are equal to 1,2,3.

Several kinematic fields are introduced: the deformation tensor $\gamma_{i j}$ - which is split into its symmetric $\gamma_{(i j)]}=\varepsilon_{i j}$ and antisymmetric part $\gamma_{[i j]}$ - and the curvature tensor $\kappa_{i j}$ - also split into split its symmetric $\kappa_{(i j)}$ and antisymmetric part $\kappa_{[i j]}$.

$$
\begin{array}{r}
\varepsilon_{i j}=\frac{1}{2}\left(u_{i, j}+u_{j, i}\right) \quad \Omega_{i j}=\frac{1}{2}\left(u_{i, j}-u_{j, i}\right) \\
\gamma_{[i j]}=\Omega_{i j}-\omega_{i j}^{c} \quad \kappa_{i j}=\omega_{i, j}^{c} \\
\gamma_{i j}=\varepsilon_{i j}+\gamma_{[i j]}=u_{i, j}-\omega_{i j}^{c}=u_{i, j}+e_{i j k} \omega_{k}^{c}
\end{array}
$$

The macroscopic strain and rotation tensors $\left(\varepsilon_{i j}\right.$ and $\left.\Omega_{i j}\right)$ are obtained as the symmetric and antisymmetric part of the displacement gradient as in the classical 50 Cauchy continuum. $\gamma_{[i j]}$ is the difference between the global rotation $\Omega_{i j}$ and the rotation of the microstructure $\omega_{i j}^{c}$. The curvature $\kappa_{i j}$ is defined as the gradient of the vector of Cosserat rotations and represents the gradient of microscopic deformations.

Correspondingly the stress tensor $\tau_{i j}$ is also divided into its symmetric $\sigma_{i j}$ 55 and antisymmetric part $\tau_{[i j]}$. The symmetric part corresponds to the macroscopic stresses (the ones that are considered in Cauchy continuum). Besides the stress tensor, a couple stress tensor $\mu_{i j}$ is introduced which is energy conjugate to the curvature.

Neglecting the volumetric forces acting on the medium and inertia terms, the 60 momentum balance equations are written as follows (Stefanou et al, 2017):

$$
\begin{aligned}
\tau_{i j, j} & =0 \\
\mu_{i j, j}-e_{i j k} \tau_{j k} & =0
\end{aligned}
$$




\subsection{Constitutive equations for a Cosserat continuum}

The general constitutive equations for a linear isotropic elastic Cosserat continuum are defined by 6 coefficients: the two classical deformation moduli, $K$ and $G$, and four new coefficients $L, M, M_{c}$ and $G_{c}$ (Mindlin, 1964). The effective stress tensor

$\tau_{i j}^{\prime}$ and couple stress tensor $\mu_{i j}$ can be expressed as:

$$
\tau_{i j}^{\prime}=C_{i j k l}^{e} \gamma_{k l}^{e} \quad \text { and } \quad \mu_{i j}=M_{i j k l}^{e} \kappa_{k l}^{e}
$$

The effective stress tensor is linked to the total stress tensor by $\tau_{i j}^{\prime}=\tau_{i j}+p \delta_{i j}$. $C_{i j k l}^{e}$ is the elastic stiffness tensor and $M_{i j k l}^{e}$ the elastic flexural bending rigidity tensor:

$$
\begin{aligned}
M_{i j k l}^{e}=\left(L-\frac{2}{3} M\right) \delta_{i j} \delta_{k l} & +\left(M+M_{c}\right) \delta_{i k} \delta_{j l} \\
+ & \left(M-M_{C}\right) \delta_{i l} \delta_{j k} \\
C_{i j k l}^{e}=\left(K-\frac{2}{3} G\right) \delta_{i j} \delta_{k l} & +\left(G+G_{c}\right) \delta_{i k} \delta_{j l} \\
+ & \left(G-G_{c}\right) \delta_{i l} \delta_{j k}
\end{aligned}
$$

For an isotropic Cosserat continuum, we introduce 6 coefficients. 3 of them have the dimensions of stress $\left(K, G\right.$ and $\left.G_{c}\right)$ and the other 3 the dimensions of length squared times stress $\left(L, M\right.$ and $\left.M_{c}\right)$. Thus, any ratio of $L, M$ or $M_{c}$ to $K, G$ or $G_{c}$ will introduce a material parameter with dimension of length squared (Cowin, 1970). For geomaterials, we usually consider $R=\sqrt{\frac{M}{G}}$ as a characteristic length related to the microstructure of the material (Unterreiner, 1994). Moreover, we 75 assume that $M=M_{c}$ to ensure that no out-of-plane couple stresses are produced during the shearing of a layer (invariance in $x_{1}$ and $x_{3}$ directions).

A flow theory of plasticity for granular media with Cosserat microstructure can be derived by keeping the same definitions for the yield surface and the plastic potential as in the classical theory, but by generalizing the stress and strain invariants to Cosserat continua (Mühlhaus and Vardoulakis, 1987). We decompose the deformation and curvature rate tensors into elastic, plastic and thermal parts (small strains theory):

$$
\dot{\gamma}_{i j}=\dot{\gamma}_{i j}^{e}+\dot{\gamma}_{i j}^{p}+\dot{\gamma}_{i j}^{t h} \quad \text { and } \quad \dot{\kappa}_{i j}=\dot{\kappa}_{i j}^{e}+\dot{\kappa}_{i j}^{p}
$$

The thermal strain rate is $\dot{\gamma}_{i j}^{t h}=\alpha \dot{T} \delta_{i j}$ with $\alpha$ the coefficient of thermal expansion. The effect of temperature on the curvature tensor is neglected herein.

The generalized deviatoric strain and stress second invariants are generalized as:

$$
\begin{array}{r}
\tau=\sqrt{h_{1} s_{i j} s_{i j}+h_{2} s_{i j} s_{j i}+\frac{1}{R^{2}}\left(h_{3} m_{i j} m_{i j}+h_{4} m_{i j} m_{j i}\right)} \\
\dot{\gamma}_{p}=\sqrt{g_{1} \dot{e}_{i j}^{p} \dot{e}_{i j}^{p}+g_{2} \dot{e}_{i j}^{p} \dot{e}_{j i}^{p}+R^{2}\left(g_{3} \dot{k}_{i j}^{p} \dot{k}_{i j}^{p}+g_{4} \dot{k}_{i j}^{p} \dot{k}_{j i}^{p}\right)}
\end{array}
$$

where $s_{i j}, m_{i j}, e_{i j}$ and $k_{i j}$ are the deviatoric parts of the effective stress, couple- 
by micro-mechanical considerations (Vardoulakis and Sulem, 1995; Rattez et al, 2018a). With the above definition of the second deviatoric stress invariants, a Drucker Prager plasticity model can be derived as in Mühlhaus and Vardoulakis (1987), or a von Mises plasticity model as in de Borst (1991).

We consider a porous medium consisting of two phases, i.e. the solid skeleton and the pore fluid.

The mass balance of the fluid phase leads to the following form of the equation for the pore pressure $p$ :

$$
\frac{\partial p}{\partial t}=c_{h y} p_{, i i}+\frac{\lambda^{*}}{\beta^{*}} \frac{\partial T}{\partial t}-\frac{1}{\beta^{*}} \frac{\partial \varepsilon_{v}}{\partial t}
$$

90 where $c_{h y}=\chi /\left(\eta^{f} \beta^{*}\right)$ is the hydraulic diffusivity, $\beta^{*}=n \beta^{f}+(1-n) \beta^{s}$ is the mixture compressibility, $\lambda^{*}=n \lambda^{f}+(1-n) \lambda^{s}$ is the coefficient of thermal expansion of the soil-water mixture (Vardoulakis, 1986). $\chi$ is the intrinsic permeability of the porous medium, and $\eta^{f}$ is the viscosity of the pore fluid. $\beta^{f}$ and $\beta^{s}$ are the compressibilities per unit volume of the pore fluid and the solid phase respectively

95 and $\lambda^{f}, \lambda^{s}$ are the thermal expansivities per unit volume. $n$ is the eulerian porosity of the medium.

\subsection{Energy balance equation}

The energy balance equation yields the following form of the evolution equation for the temperature $T$ :

$$
\rho \mathrm{C}\left(\frac{\partial T}{\partial t}-c_{t h} T_{, i i}\right)=\tau_{i j}^{\prime} \dot{\gamma}_{i j}^{p}+\mu_{i j} \dot{\kappa}_{i j}^{p}
$$

where $c_{t h}=\frac{k_{T}}{\rho \mathrm{C}}$ is the thermal diffusivity, $k_{T}$ is the thermal conductivity of the material, $\rho \mathrm{C}$ is the specific heat per unit volume of the material in its reference state.

\section{Finite element implementation and validation tests}

In this section, the numerical implementation of a Cosserat continuum with THM couplings is presented and tested through a series of simulations based on various benchmarks for the different physical couplings. The tests are summarized in this section. 
3.1 Numerical implementation

A displacement-rotation-temperature-pore pressure incremental finite element formulation is used to numerically integrate the model presented in the previous section. The integration in time is implicit using the backward Euler method, which is implemented in REDBACK (Poulet et al, 2016).

The weak form of the linear and angular momentum balance equations Eqs. 3-4 is written as (Godio et al, 2015):

$$
\begin{aligned}
-\int_{\Omega} \tau_{i j} \psi_{i, j} d \Omega+\int_{\partial \Omega_{\Sigma}} \tau_{i j} n_{j} \psi_{i} d S & =0 \\
-\int_{\Omega} \mu_{i j} \psi_{i, j} d \Omega+\int_{\partial \Omega_{\Sigma}} \mu_{i j} n_{j} \psi_{i} d S-\int_{\Omega} \varepsilon_{i j k} \tau_{j k} \psi_{i} d \Omega & =0
\end{aligned}
$$

The energy and fluid mass balance equations are also written in their weak forms:

$$
\begin{aligned}
& \int_{\Omega} \dot{p} \psi d \Omega+c_{h y}\left(\int_{\Omega} p_{, i} \psi, i d \Omega-\int_{\partial \Omega} p_{, i} n_{i} \psi d S\right)-\frac{\lambda^{*}}{\beta^{*}} \int_{\Omega} \dot{T} \psi d \Omega+\frac{1}{\beta^{*}} \int_{\Omega} \dot{\varepsilon_{v}} \psi d \Omega=0 \\
& \int_{\Omega} \dot{T} \psi d \Omega+c_{t h}\left(\int_{\Omega} T_{, i} \psi, i d \Omega-\int_{\partial \Omega} T_{, i} n_{i} \psi d S\right)-\frac{1}{\rho C} \int_{\Omega}\left(\tau_{i j}^{\prime} \dot{\gamma}_{i j}^{p}+\mu_{i j} \dot{\kappa}_{i j}^{p}\right) \psi, i d \Omega=0
\end{aligned}
$$

where $\psi$ and $\psi_{i}$ are tests functions. $c_{h y}, c_{t h}, \rho \mathrm{C}, \beta^{*}$ and $\lambda^{*}$ are considered constant herein.

Linear Lagrange test functions are chosen for all the fields and full integration is performed. Quadratic interpolation functions could be used as well for the displacement field, linear for the Cosserat rotations and linear or constant for the pressure and temperature fields in order to assure compatible interpolation order and lead to a more efficient formulation. Moreover, reduced integration could be also used to improve performance (see (Godio et al, 2015) for a detailed discussion). However, the mesh convergence analyses performed herein, show acceptable performance and convergence despite the use of linear shape functions for all the fields.

\subsection{Mechanical tests}

As a first step, the implementation of the equations for a Cosserat continuum and of the constitutive laws are checked. For elasticity, the numerical results are compared to the analytical solution of shearing of an elastic layer and for plasticity with results found in the literature.

\subsubsection{Elasticity tests}

The numerical results are compared with an analytical solution called the bound- 
We consider the shearing of an elastic infinite layer of thickness $h$ in plane strain conditions. The geometry is described in Fig. 2.

We use the stress-strain relationships of a 2D-linear isotropic elastic Cosserat medium in plane strain conditions.

All mechanical properties are assumed to be independent from the $x_{1}$ coordinates, which results in $\gamma_{11}=0$ and $\kappa_{31}=0$. We also assume that $\tau_{22}=0$ at the top and $u_{2}=0$ at the bottom, which results in $\tau_{11}=\tau_{22}=\gamma_{22}=0$ everywhere in the layer.

In that case, the equilibrium equations can be reduced to only two equations:

$$
\begin{aligned}
& \frac{\partial \tau_{12}}{\partial x_{2}}=0 \quad \text { or } \quad \tau_{12}=\text { constant }=\tau_{c} \\
& \frac{\partial \mu_{32}}{\partial x_{2}}+\tau_{21}-\tau_{12}=0
\end{aligned}
$$

Then, we insert the constitutive equations to obtain a coupled system of partial differential equations in $u_{1}$ and $\omega_{3}^{c}$ :

$$
\begin{gathered}
\left(G+G_{c}\right) \frac{\partial^{2} u_{1}}{\partial x_{2}^{2}}+2 G_{c} \frac{\partial \omega_{3}^{c}}{\partial x_{2}}=0 \\
M \frac{\partial^{2} \omega_{3}^{c}}{\partial x_{2}^{2}}-4 G_{c} \omega_{3}^{c}=2 G_{c} \frac{\partial u_{1}}{\partial x_{2}}
\end{gathered}
$$

The general solution of this system is:

$$
\begin{array}{r}
\omega_{3}^{c}=K_{1} e^{\delta \cdot x_{2}}+K_{2} e^{-\delta \cdot x_{2}}-\frac{\tau_{c}}{2 G} \\
u_{1}=-\frac{2 G_{c}}{G+G_{c}}\left(\frac{K_{1}}{\delta} e^{\delta \cdot x_{2}}-\frac{K_{2}}{\delta} e^{-\delta \cdot x_{2}}\right)+\frac{\tau_{c}}{G} x_{2}+K_{3}
\end{array}
$$

where $\delta=2 \sqrt{\frac{G G_{c}}{\left(G+G_{c}\right) M}}$

The four constants $\left(K_{1}, K_{2}, K_{3}\right.$ and $\left.\tau_{c}\right)$ are determined from the boundary conditions in $u_{1}$ and $\omega_{3}^{c}$.

At the bottom of the layer $\left(x_{2}=0\right)$, the displacement is equal to 0 and the Cosserat rotation is constrained to be equal to the rigid body rotation.

$$
u_{1}\left(x_{2}=0\right)=0 \quad \text { and } \quad \omega_{3}^{c}\left(x_{2}=0\right)=\Omega_{21}\left(x_{2}=0\right)=-\frac{1}{2} \frac{\partial u_{1}}{\partial x_{2}}\left(x_{2}=0\right)
$$

At the top of the layer $\left(x_{2}=h\right)$, the displacement is imposed equal to $0.01 \mathrm{~mm}$ and the Cosserat rotation is equal to -0.1. It corresponds to an interface with a particular roughness (Vardoulakis et al, 1992; Vardoulakis and Unterreiner, 1995).

$$
u_{1}\left(x_{2}=h\right)=0.01 \mathrm{~mm} \text { and } \omega_{3}^{c}\left(x_{2}=0\right)=-0.1
$$

The set of parameters chosen as an example are given in Table 1.

We choose a characteristic length $R$ (or bending length) one order of magnitude less than the thickness of the band and we observe that the Cosserat effects are confined in a layer of size about three times $R$ adjacent to the upper boundary, where the rotation and displacement are imposed.

For the geometry of the numerical simulation, we consider a cube of dimensions $1^{*} 1^{*} 1 \mathrm{~mm}^{3}$ with 50 elements along $x_{2}$-direction, 10 along $x_{1}$-direction and 1 along 


\begin{tabular}{|c|c|c|}
\hline Parameters & values & units \\
\hline$G$ & 10 & $\mathrm{GPa}$ \\
$G_{C}$ & 20 & $\mathrm{GPa}$ \\
$h$ & $10^{-3}$ & $\mathrm{~m}$ \\
$R$ & $10^{-4}$ & $\mathrm{~m}$ \\
$M$ & $G R^{2}$ & $\mathrm{kN}$ \\
\hline
\end{tabular}

Table 1 Values of the parameters used for the boundary layer elastic test

$x_{3}$-direction. We prescribe periodic boundaries in the $x_{1}$ - and $x_{3}$-directions. We have 6 variables in our model $\left(u_{1}, u_{2}, u_{3}, \omega_{1}^{c}, \omega_{2}^{c}, \omega_{3}^{c}\right)$, thus their values or the values of their derivatives have to be imposed at the top and bottom edges.

Bottom edge $\left(x_{2}=0\right)$ :

$$
u_{1}=0 ; \quad u_{2}=0 ; \quad u_{3}=0 ; \quad \omega_{1}^{c}=0 ; \quad \omega_{2}^{c}=0 ; \quad \omega_{3}^{c}=\Omega_{21}
$$

Top edge $\left(x_{2}=h\right)$ :

$$
\frac{u_{1}}{h}=0.01 ; \quad \frac{\partial u_{2}}{\partial x_{2}}=0 ; \quad u_{3}=0 ; \quad \omega_{1}^{c}=0 ; \quad \omega_{2}^{c}=0 ; \quad \omega_{3}^{c}=-0.1
$$

In Fig. 3(a), the results for the displacements are plotted on the deformed mesh. In Figs. 3(b), we compare the values obtained for $\omega_{3}^{c}$ with the analytical solution presented above. The difference between the numerical and the analytical solutions is negligible. Moreover, a mesh convergence analysis is conducted for the value of the Cosserat rotation at $x_{2}=0.9$ (see Fig. 4), which shows that the relative error is less than $1.2 \%$ for 40 elements and decreases to $0.01 \%$ for 400 elements (see also Godio et al (2015) for a mesh convergence in terms of modal frequencies with Cosserat continuum).

\subsubsection{Plasticity tests}

The Drucker-Prager plastic model for three dimensional Cosserat continua is developed in Rattez et al (2018a) assuming a hardening law arising from the evolution of the friction coefficient. In order to compare with results in the literature that consider a von Mises yield criterion, we assume the following expression for the yield criterion:

$$
\begin{aligned}
& F=\tau+\mu \sigma\left(1+h_{s} q \xi\right)-c\left(1+h_{s} q(1-\xi)\right) \\
& Q=\tau+\beta \sigma\left(1+h_{s} q \xi\right)-c\left(1+h_{s} q(1-\xi)\right)
\end{aligned}
$$

where $\sigma=\frac{\tau_{k k}}{3}$ and $\tau$ is the generalized second invariant of the deviatoric stresses. $\xi$ is a parameter that allows us either to have a hardening on the friction coefficient $(\xi=1)$ as for the Drucker-Prager model derived in Rattez et al (2018a), or on the cohesion $(\xi=0)$ as for the von Mises yield criterion. $h_{s}$ is a parameter controlling the magnitude of the hardening/softening rate and $q$ is the hardening variable (chosen as $\dot{q}=\dot{\gamma}^{p}$ ).

As an example, we consider an infinite layer of height $h=60 \mathrm{~mm}$ subjected to pure shear at constant velocity $V$ (see Fig. 5 ). The same material parameters with Godio et al (2016) are chosen in order to have a comparison. 
The yield criterion is based on a von Mises model adapted for Cosserat continua, as in de Borst et al (2012). In equation 26, we consider $\xi=0, \mu=0$ in order to have the same expression.

$$
F=\tau-c\left(\gamma^{p}\right)
$$

where $c$ is the equivalent plastic stress (or cohesion) that depends linearly on $\gamma^{p}$ the equivalent plastic strain. The coefficients in the stress invariant are $h_{1}=h_{2}=\frac{3}{4}$, $h_{3}=\frac{3}{2}$ and $h_{4}=0$. In the plastic strain invariant they are $g_{1}=g_{2}=\frac{1}{3}, g_{3}=\frac{2}{3}$ and $g_{4}=0$. These values for the coefficients differ from Mühlhaus and Vardoulakis (1987); Mühlhaus (1986); Vardoulakis and Sulem (1995), because of the use of a von Mises criterion for which yielding occurs when $\tau$ reaches the yield strength of the material in simple tension, leading to the conditions $h_{1}+h_{2}=\frac{3}{2}$ and $g_{1}+g_{2}=\frac{2}{3}$. In the other papers, yielding is chosen to occur when $\tau$ reaches the yield strength of the material in pure shear, leading to the conditions $h_{1}+h_{2}=\frac{1}{2}$ and $g_{1}+g_{2}=2$.

In this purely mechanical example, a linear softening rule is chosen to exhibit a localization of the deformations. The initial cohesion is $c=100 \mathrm{MPa}$ and the hardening modulus is $h_{s}=-4$.

The elastic properties of the material are $K=4000 \mathrm{MPa}, G=4000 \mathrm{MPa}$ and $G_{c}=2000 \mathrm{MPa}$. The internal length of the microstructured continuum is chosen to be $R=2.5 \mathrm{~mm}$.

We investigate, first, the mesh-convergence of the model, which is the one of the most interesting features of the Cosserat continua. A three-dimensional geometry is considered with periodic boundary conditions for the right, left, front and back side of the cube. A regular mesh is chosen with one element in directions $x_{1}$ and $x_{3}$, and a range of 32 to 300 in the direction $x_{2}$. In Fig. $6(\mathrm{~b})$, the shear stress $\tau_{12}$ at the top of the layer is plotted versus the normalized horizontal displacement at the top. The plastic regime is reached for $\tau_{12}=\frac{c}{\sqrt{3}}$ as expected and shows a softening behavior, exactly like Godio et al (2016) and de Borst (1991). The results for 200 and 300 elements in the vertical direction exhibit no clear difference, showing a mesh-convergence.

In Fig. 6(a), the Cosserat rotation around direction $x_{3}$ is plotted on the deformed mesh for $\tau_{12}=26 \mathrm{MPa}$ after softening. The magnitude of the rotations is higher inside the zone of localized deformations as observed experimentally (Hall et al, 2010).

In Fig. 7, the shear strain $\gamma_{12}$ is plotted along the height of the layer for different discretizations and for $\tau_{12}=26 \mathrm{MPa}$ after softening. The deformation profile is practically identical for 80 and 200 elements showing also a mesh convergence.

In Fig. 8(a), we present the stress-strain evolution of the shear layer discretized with only one element. This allows to test the implementation of the hardening/softening constitutive behavior. Both the static Mühlhaus-Vardoulakis and de Borst coefficients for the shear stress and shear strain generalized invariants are used and a hardening/softening coefficient of $4 /-4 \mathrm{MPa}$ is used. We observe that the yield criterion is reached for $\tau_{12}=\frac{c}{\sqrt{3}}$ considering de Borst coefficients and $\tau_{12}=c$ considering Mühlhaus-Vardoulakis choice of coefficients.

In Fig. 8(b), we plot the generalized stress invariant $\tau$ as a function of the generalized plastic strain invariant $\gamma_{p}$. The slopes in this diagram enable us to 


\subsection{Thermo-Mechanical tests}

These tests concern the verification of the coupling between the energy balance equation and the mechanical equations (Eqs. 12, 13 and 15).

First, we look at the heating of a rod of length $10 \mathrm{~mm}$ with a section of $1 \times 1 \mathrm{~mm}^{2}$

230 (Fig. 9).

For the first test, the evolution of temperature at the middle of the rod is compared to the solution obtained analytically using series (Fig. 10(a)) (Kreyszig, 2006). A good agreement is found. In Fig. 10(b), we plot the evolution of the displacement at the free end of the rod for $c_{t h}=1 \mathrm{~mm}^{2} / \mathrm{s}$. It tends to a value of $2.5 \times 10^{-2} \mathrm{~mm}$ as expected (thermal expansion coefficient: $2.5 \times 10^{-2} /{ }^{\circ} \mathrm{C}$ ).

Additional tests are performed, for the same geometry of the rod, but with different boundary conditions. In these tests the normal displacements of all faces are fixed except on one side as shown in Fig. 11. The rod is no longer free to expand in all directions and the precluded deformations cause the development of stresses that can be calculated analytically in elasticity.

The deformation along the $x_{3}$-axis of the rod is given by:

$$
\gamma_{33}=\frac{3 K}{K+\frac{4}{3} G} \alpha \Delta T
$$

and the stresses perpendicular to the axis of the rod are:

$$
\tau_{11}=\tau_{22}=\left(K-\frac{2}{3} G\right) \gamma_{33}-3 K \alpha \Delta T
$$

Therefore, when the temperature reaches a steady state $\Delta T=1^{\circ} \mathrm{C}$, we obtain $\gamma_{33}=3.75 \times 10^{-2}$ and $\tau_{11}=\tau_{22}=-1.125 \times 10^{-2} \mathrm{MPa}$ (with $K=3 \mathrm{MPa}$ and $G=1.5 \mathrm{MPa})$. These values are in agreement with the results of the numerical simulation shown in Fig. 12.

The two last tests are dedicated to Thermo-Mechanical couplings considering an elasto-plastic behavior in order to explore the influence of a temperature rise on the plastic behavior of the material. The configuration for the first test is the one shown in Fig. 11, but this time a yield stress (cohesion) is set to $0.01 \mathrm{MPa}$. Perfect plasticity is considered in this example, but with no plastic dissipation in the energy balance equation. In Fig. 13, the results for the stress and displacement are compared. We observe that $\tau_{11}$ remains constant after reaching the value of 0.01MPa. The development of plastic deformations also has a consequence on the displacement in the rod axis direction $\left(x_{3}\right)$, which is higher than that for the elastic rod. Nevertheless, no difference is observed for the evolution of the temperature as the mechanical dissipation term is neglected in this example. Thus, no feedback between the mechanical behavior and the energy balance is possible.

In order to have an insight into the feedback of the mechanics on the energy balance equation (due to the plastic dissipation term), a shear test is modeled. The same parameters as the plastic tests with a height of $60 \mathrm{~mm}$ and only one element is considered for the mesh in the vertical direction. For simplicity, we neglect heat diffusion and only consider the effect of plastic dissipation on the temperature change:

$$
\rho \mathrm{C} \frac{\partial \mathrm{T}}{\partial t}=\sigma_{i j} \dot{\varepsilon}_{i j}^{p}+\tau_{[i j]} \dot{\gamma}_{[i j]}^{p}+\mu_{i j} \dot{\kappa}_{i j}^{p}
$$


From this test, we verify the value of the mechanical dissipation $\tau_{12} \frac{\Delta \gamma_{12}^{p}}{\Delta t}$ at each time step. Moreover, from the energy balance equation (Eq. 31), the temperature increase between two time steps in the plastic regime is equal to $\frac{\tau_{12} \Delta \gamma_{12}^{p} \Delta t}{\rho \mathrm{C}}$ (with $\rho \mathrm{C}$ taken equal to $1 \mathrm{MPa} /{ }^{\circ} \mathrm{C}$ here). The comparison of this expression and of the actual temperature increase is plotted in Fig. 14 showing no difference.

\subsection{Hydro-Mechanical tests}

Three tests have been designed in order to check the Hydro-Mechanical (HM) couplings (Eqs. 12, 13 and 14). An undrained oedometer configuration is considered. The geometry and boundary conditions are shown in Fig. 15.

No mass diffusion is considered in these tests and, therefore, the mass conservation equation becomes:

$$
\frac{\partial p}{\partial t}=-\frac{1}{\beta^{*}} \frac{\partial \varepsilon_{v}}{\partial t}
$$

A cubic single element of dimensions $1 \times 1 \times 1 \mathrm{~mm}^{3}$ representing a fully saturated sample with its lateral displacements prevented is considered. Moreover, all boundaries are impervious. A constant downward velocity $v_{3}$ is applied on the top boundary.

The deformation of the cube in the direction of the applied velocity is:

$$
\gamma_{33}=-\frac{v_{3} t}{L}
$$

The effective normal stresses are linked to the elastic deformation by:

$$
\begin{aligned}
\tau_{11}^{\prime}=\tau_{22}^{\prime} & =\left(K-\frac{2 G}{3}\right) \gamma_{33} \\
\tau_{33}^{\prime} & =\left(K+\frac{4 G}{3}\right) \gamma_{33}
\end{aligned}
$$

No shear stresses are developed and the rise in pore pressure is:

$$
p=-\frac{\gamma_{33}}{\beta^{*}}
$$

We consider first two tests with an elastic behavior for the material. The comparison of the pore pressure rise compared to the analytical solution is plotted in Fig. 16 and the two evolution match perfectly $\left(v_{3}=10^{-2} \mathrm{~mm} \cdot \mathrm{s}^{-1}\right.$ and $\left.\beta^{*}=2 \mathrm{MPa}^{-1}\right)$.

The solution in terms of total stresses is validated against analytical results is shown in Fig. 17(a).

A last test is designed for HM couplings considering an elasto-plastic behavior and allows us to test the numerical implementation of the plastic constitutive behavior and its coupling with the pore pressure. In this test a cohesion of $0.1 \mathrm{MPa}$ is considered with zero friction.

In oedometric conditions, the generalized shear stress invariant is equal to:

$$
\tau=\sqrt{\frac{2}{3}\left(h_{1}+h_{2}\right)\left(\tau_{11}^{\prime}-\tau_{33}^{\prime}\right)^{2}}
$$


Combining Eqs. 37, 34 and 3.4 the yield criterion is reached at a time:

$$
t=\frac{\sqrt{3} c L}{2 G v_{3} \sqrt{2\left(h_{1}+h_{2}\right)}}
$$

which is equal to $3.33 \mathrm{~s}$ for the parameters considered here $\left(G=1.5 \mathrm{MPa}, v_{3}=\right.$ $10^{-2} \mathrm{~mm} \cdot \mathrm{s}^{-1}$ and de Borst coefficients in the invariant). This value is compatible with the numerical results presented in Fig. 17(b).

\subsection{Thermo-Hydro-Mechanical tests}

In order to test the implementation of THM couplings, we consider a shear test taking into account the full set of equations of our model (Eqs. 14, 15, 12 and 13). Similar to the shearing test for Thermo-Mechanical couplings, a height of $60 \mathrm{~mm}$ with only one element in the vertical direction is considered. Assuming no heat and fluid flux (undrained adiabatic case), we obtain:

$$
\frac{\partial p}{\partial t}=\frac{\lambda^{*}}{\beta^{*}} \frac{\partial T}{\partial t}
$$

In this test, we focus on the thermal pressurization term in the mass balance equation. The coefficient $\frac{\lambda^{*}}{\beta^{*}}$ is taken equal to $0.5 \mathrm{MPa} /{ }^{\circ} \mathrm{C}$. The results for the pressure and temperature are plotted in Fig. 18. We can see that there is a ratio of $0.5 \mathrm{MPa} /{ }^{\circ} \mathrm{C}$ between the two graphs, which corresponds to the value of the thermal pressurization coefficient. We observe here that the plastic dissipation induces an increase of temperature, which leads to a pore pressure increase (thermal pressurization).

\section{Examples of numerical simulations}

In order to illustrate the capabilities of the proposed model in geomechanical applications, two different problems are considered in this section. The first one is a standard biaxial test for a weak sandstone. The second is an infinite sheared layer modeling a mature fault zone in the brittle part of the lithosphere.

\subsection{Biaxial test for sandstones}

Sandstones are sedimentary rocks formed by cemented sand grains. These grains present diameters within the range of $0.0625 \mathrm{~mm}$ to $2 \mathrm{~mm}$ according to UddenWentworth particle size classification (Wentworth, 1922). Due to this microstructure, one of the main deformation mechanisms observed in sandstones is a granular flow characterized by grain sliding and rolling (Fossen et al, 2007). Therefore, a Cosserat continuum appears to be an appropriate framework to describe this class of materials. Moreover, because of their generally high porosity and permeability, many of the oil, gas, and groundwater reservoirs in the world are found in sandstones (Bjorlykke, 2010). The study of deformation bands is thus of primary 
importance for hydrogeologists due to hydraulic properties modifications, which usually accompany their development (e.g. permeability reduction) and can act as barriers or channels to fluid flow.

The conditions of temperature and pore pressure at the depth of the reservoirs influence their mechanical behavior. A Thermo-Hydro-Mechanical model is necessary to capture correctly the behavior of the medium at depth.

In this study, we consider a Drucker-Prager yield surface that can describe the behavior of geomaterials that exhibit dilatancy when sheared like sandstones at low confining pressures. It should be noted that this yield surface is not suitable to describe compactional shear bands formed at higher confining pressures (Stefanou and Sulem, 2014a). In this case, a yield surface presenting a cap at high mean stresses is necessary (Issen and Rudnicki, 2000).

We consider a biaxial geometry and the mechanical parameters are derived from triaxial compression test carried in Castlegate sandstone and described in Zervos et al (2001). The geometry and the boundary conditions are shown in Fig. 19. The specimen has a height of $10 \mathrm{~cm}$ and a width of $5 \mathrm{~cm}$. A displacement is progressively imposed at the top boundary with a constant velocity. The normal displacement at the bottom boundary are precluded as well as the horizontal displacement of the point at the bottom right corner.

A Drucker-Prager yield surface is chosen as it adequately captures the behavior of the sandstone at shearing.

$$
F=\tau+\mu \sigma^{\prime}-c
$$

The coefficients in the generalized stress invariant $\tau$ are $h_{1}=h_{3}=\frac{2}{3}$ and $h_{2}=h_{4}=-\frac{1}{6}$. The coefficients the generalized plastic strain invariant $\gamma^{p}$ are $g_{1}=g_{3}=\frac{8}{5}$ and $g_{2}=g_{4}=\frac{2}{5}$. The cohesion $c$ evolves with the equivalent plastic strain following a hyperbolic law (Zervos et al, 2001):

$$
c=c_{0}+\frac{\left(1-K_{1} \gamma^{p}\right) \gamma^{p}}{K_{2}+K_{3} \gamma^{p}}
$$

where $K_{2}$ and $K_{3}$ are coefficients calibrated against experimental results, $K_{2}=$ $1.323 \times 10^{-5}$ and $K_{3}=6.1271 \times 10^{-2} . c_{0}$ is a threshold value for the cohesion that governs the initiation of the plastic behavior, taken here equal to $6.25 \mathrm{MPa} . K_{1}$ is an open parameter that defines the hardening (or softening) rate of the material. Its influence on the stress-strain response is shown in Fig. 21.

Based on micromechanical considerations that enable to link the internal lengths of Cosserat continua to the mean grain size of a granular medium (e.g. (Rattez et al, 2018a)), it is possible to investigate qualitatively the influence of the characteristic size of the microstructure on the overall stress-strain response of the system and the shear band thickness. This is accomplished by varying the representative mean grain size, as shown in Fig. 22.

The softening behavior of the stress-strain diagram is more pronounced for smaller grains as the shear band tends to be thinner. The elastic part and the hardening part of the diagrams are not affected by the grain size. The difference in the shear band size is illustrated in Fig. 23, where the generalized plastic strain invariant is plotted on the deformed mesh for the same value of the shear stress 
$\left(\tau_{12}=40 \mathrm{MPa}\right.$ after softening). In Rattez et al (2018b), the shear band size is evaluated using the plastic strain rate $\gamma^{p}$ as a proxy, because it allows an instantaneous evaluation of the localization process ( $\gamma^{p}$ depends on the deformation history). Therefore, a thinner plastic zone as obtained in the case of the smaller grain size corresponds to the specimen in which a thinner zone of localized deformation has formed.

In order to trigger the localization, an imperfection has to be introduced. In this paper, a notch is introduced at the bottom left of the specimen. This enables to have the same deformation pattern in all the tests and to obtain stress-strain diagrams that can be compared (Besuelle et al, 2006).

The effect of the THM couplings on the response of the material is shown in Fig. 24. The test taking into account only the mechanical equations corresponds to an isothermal and drained material. In the THM model, we consider the feedback of the evolution of the temperature and pore pressure on the mechanical behavior. We observe softening due to the thermal pressurization phenomenon, but it is not very pronounced as the plastic strain remains small and the magnitude of temperature and pore pressure fields are not high enough to strongly affect the mechanical response. However, in the case of the shearing of an infinite layer modeling a fault zone, the effect of thermal pressurization is much more significant as described in the next section.

\subsection{Shearing of a fluid saturated mature fault zone}

Observations of faults from drilling or outcrops show the formation of a slip zone of finite but very small thickness, composed of cataclastic material due to excessive shearing, called gouge material (Sibson, 2003). These zones are of primary importance as they accommodate most of the slip during a seismic event. Further investigations indicate that an even thinner zone of ultracataclastic material, called the Principal Slip Zone (PSZ) exists inside the gouge (Rice, 2006a). The thickness of these shear bands is a key parameter for understanding fault behavior (Kanamori and Brodsky, 2004) as it is related to the triggering and evolution of various multiphysical couplings and to the energy dissipation during seismic slip.

A substantial grain size reduction (grain cataclasis) is usually used as a marker to identify the principal slip zone (Sammis and Ben-Zion, 2008). Moreover, many physical processes are directly affected by grain size evolution. First of all, the production of fine particles can significantly weaken the material. Previous studies have shown that the production of nanograins during slip reduces the friction coefficient due the lubrication of the slip surface (Han et al, 2010; Di Toro et al, 2011). The grain size affects the frictional properties (Anthony and Marone, 2005) and the strength of the gouge (Dieterich, 1981). Furthermore, grain size also has an effect on the dissolution or dehydration reactions occuring in faults as chemical reactions kinetics depend on the specific surface (Hu and Hueckel, 2007; Stefanou and Sulem, 2014b). Therefore, it is necessary to include information about the size of the microstructure and its evolution in the constitutive model of a gouge.

In order to describe the influence of weakening processes and of the size of the microstructure in a gouge during a seismic event, a THM model of a fault gouge is developed in this section. The fault zone is modeled here as a saturated infinite 


\begin{tabular}{|c|c|c||c|c|c|}
\hline parameters & values & units & parameters & values & units \\
\hline$K$ & $20 \times 10^{3}$ & $\mathrm{MPa}$ & $\mu$ & 0.5 & \\
$G$ & $10 \times 10^{3}$ & $\mathrm{MPa}$ & $\beta$ & 0 & \\
$G c$ & $5 \times 10^{3}$ & $\mathrm{MPa}$ & $\lambda^{*}$ & $7.4 \times 10^{-5}$ & $/{ }^{\circ} \mathrm{C}$ \\
$R$ & 10 & $\mu \mathrm{m}$ & $\rho C$ & 2.8 & $\mathrm{MPa} /{ }^{\circ} \mathrm{C}$ \\
$\rho$ & 2500 & $\mathrm{~kg} / \mathrm{m}^{3}$ & $c_{t h}$ & 1 & $\mathrm{~mm}^{2} / \mathrm{s}$ \\
$\beta^{*}$ & $8.2 \times 10^{-5}$ & $\mathrm{MPa}$ & $c_{h y}$ & 12 & $\mathrm{~mm}^{2} / \mathrm{s}$ \\
$n$ & 0.04 & & $\alpha$ & $2.5 \times 10^{-5}$ & $/{ }^{o} \mathrm{C}$ \\
\hline
\end{tabular}

Table 2 Numerical values for the parameters of a deep rock gouge from (Sulem et al, 2011; Rice, 2006b).

layer sheared at its top with a constant velocity of $1 \mathrm{~m} / \mathrm{s}$, a typical rate reached during a seismic slip.

The values of the different parameters are retrieved from Rattez et al (2017) and Rice (2006a) for a gouge material lying at $7 \mathrm{~km}$, a centroidal depth for crustal faults. These values are summarized in Table 2. Considering the great depth of the fault, zero dilatancy is assumed here (cf. Sleep (1999)).

In order to emphasize the role of the softening due to thermal pressurization, perfect plasticity is considered (Fig. 25). For the shearing of a gouge considering only the mechanical equations, no softening is observed as expected. Whereas with THM couplings a significant decrease of the shear stress appears in the plastic regime. However, it requires a sufficient accumulated slip before a notable THM softening takes place. With this set of parameters, for a decrease of $10 \%$ of the shear stress, a shear deformation of $30 \%$ of the layer is necessary.

The hydraulic parameters of fault gouges are poorly constrained (Segall and Rice, 2006) and are central in the behavior of a fault. For instance, the thermal pressurization weakening is magnified by the capacity of the porous space to trap a pressurized heated pore fluid. If the fluid can diffuse in the surrounding medium, the pressurization is precluded and cannot operate efficiently causing the instability propagation to terminate (Wibberley et al, 2008). Thus, we investigate the influence of the permeability on the response of the gouge material in Fig. 26.

The permeability of fault zones depends on many parameters such as the rock type, the stress state, the fault architecture, among others (Wibberley and Shimamoto, 2003). Moreover, its value is not constant along the fault and evolve during shearing (Sulem and Famin, 2009). A parametric study on this parameter is consequently conducted for typical values ranging from $10^{-17} \mathrm{~m}^{2}$ to $10^{-19} \mathrm{~m}^{2}$ in Fig. 26. For higher permeabilities, the softening is less severe as the shear band is thicker. Indeed, the diffusion of the pore fluid tends to enlarge the band. This outcome is illustrated in Fig. 27. The deformed mesh for two different permeabilities are plotted for the same level of deformation and exhibit different shear band thicknesses. For lower permeabilities the pressurization inside the shear band is stronger enhancing softening (see also Scuderi and Collettini (2016) for recent experimental evidence).

\section{Concluding Remarks}

A numerical model for Thermo-Hydro-Mechanical (THM) couplings in Cosserat continua is presented, implemented and validated in this paper. The fully coupled 
nonlinear set of equations is integrated using an incremental finite element solver. Notice that staggered (sequential) schemes may lead to peculiar numerical artifacts and instabilities.

The implementation is thoroughly tested in order to certify the results obtained afterwards. The proposed tests can be used also as guidelines for implementations of other THM codes. A particular attention is given to the coupling terms related to temperature and pore pressure evolution and their impact on the mechanical behavior.

Thereafter, the numerical tool is used to study biaxial tests for sandstones using a hyperbolic law to describe their softening. The influence of the size of the microstructure on the shear band thickness and, as a result, on the stress-strain diagram is shown. However, as the range of deformation of these tests remains limited, the thermal pressurization weakening effect is rather small.

Finally, the problem of a sheared saturated mature fault zone is studied. A perfect plastic law is introduced to exhibit clearly the role of the THM couplings on the softening behavior of the material, which are significant. A parametric study on the role of the permeability is carried out and highlights the major role played by the diffusion processes on the strain localization phenomenon.

Acknowledgements The second author, I.S., would like to acknowledge support of the European Research Council (ERC) under the European Unions Horizon 2020 research and innovation program (grant agreement No 757848 CoQuake).

\section{References}

Anthony JL, Marone CJ (2005) Influence of particle characteristics on granular friction. Journal of Geophysical Research B: Solid Earth 110(8):1-14, DOI 10.1029/2004JB003399

Besuelle P, Chambon R, Collin F (2006) Switching deformations modes in postlocalization solutions with a quasi-brittle material. journal of materials and structures 1(7):1115-1134

Bjorlykke K (2010) Petroleum Geoscience: from Sedimentary Enviroments to Rock Physics. DOI 10.1007/978-3-642-02332-3, arXiv: 1011.1669v3

de Borst R (1991) Simulation of Strain Localization: a Reappraisal of the Cosserat Continuum. Engineering Computations 8(4):317-332, DOI 10.1108/eb023842

475 de Borst R, Crisfield MA, Remmers JJC, Verhoosel CV (2012) Nonlinear Finite Element Analysis of Solids and Structures. John Wiley \& Sons

Cowin SC (1970) Stress Functions for Cosserat Elasticity. International Journal of Solids and Structures 6(4):389-398

Di Toro G, Han R, Hirose T, De Paola N, Nielsen S, Mizoguchi K, Ferri F, Cocco M, Shimamoto T (2011) Fault lubrication during earthquakes. Nature 471(7339):494-498, DOI 10.1038/nature09838, URL http://www . nature.com/doif inder/10.1038/nature09838

Dieterich JH (1981) Constitutive properties of faults with simulated gouge. Geophysical Monograph Series 24:103-120, URL

485 http://www.agu.org/books/gm/v024/GM024p0103/GM024p0103.shtml

Fossen H, Schultz RA, Shipton ZK, Mair K (2007) Deformation bands in sandstone: a review. Journal of the Geologi- 
cal Society 164(4):755-769, DOI 10.1144/0016-76492006-036, URL http://jgs.lyellcollection.org/cgi/doi/10.1144/0016-76492006-036

Godio M, Stefanou I, Sab K, Sulem J (2015) Dynamic finite element formulation for Cosserat elastic plates. International Journal for Numerical Methods in Engineering 101(13):992-1018, DOI 10.1002/nme.4833〉, URL https://hal .archives-ouvertes.fr/hal-01114343

Godio M, Stefanou I, Sab K, Sulem J (2016) Multisurface plasticity for Cosserat materials: plate element implementation and validation. International Journal for Numerical Methods in Engineering DOI 10.1002/nme.5219

Goodman RE (1989) Introduction to rock mechanics. John Wiley \& Sons, Inc., DOI 10.1016/0148-9062(81)90521-0, URL http://linkinghub.elsevier.com/retrieve/pii/0148906281905210, 9809069v1

Hall SA, Bornert M, Desrues J, Lenoir N, Pannier Y, Viggiani G, Bésuelle P (2010) Discrete and continuum analysis of localised deformation in sand using $\mathrm{X}$-ray $\mu \mathrm{CT}$ and volumetric digital image correlation. Géotechnique 60(5):315322, DOI 10.1680/geot.2010.60.5.315

5 Han R, Hirose T, Shimamoto T (2010) Strong velocity weakening and powder lubrication of simulated carbonate faults at seismic slip rates. Journal of Geophysical Research: Solid Earth 115(3), DOI 10.1029/2008JB006136

$\mathrm{Hu}$ LB, Hueckel T (2007) Coupled chemo-mechanics of intergranular contact: Toward a three-scale model. Computers and Geotechnics 34(4):306-327, DOI 10.1016/j.compgeo.2007.02.009

Issen KA, Rudnicki JW (2000) Conditions for compaction bands in porous rock. Journal of Geophysical Research: Solid Earth 105(B9):21,529, DOI 10.1029/ 2000JB900185, URL http://doi.wiley.com/10.1029/2000JB900185

Kanamori H, Brodsky EE (2004) The physics of earthquakes. Reports on Progress in Physics 67(8):1429-1496, DOI 10.1088/0034-4885/67/8/R03

Kreyszig E (2006) Advanced Engineering Mathematics, 9th edn. Wiley, New York, DOI 10.1017/CBO9781107415324.004, URL https : //books . google.com/books?id=diBo7dXRLwoC $\{\&\}$ pgis=1, arXiv: $1011.1669 \mathrm{v} 3$

Longuemare P, Mainguy M, Lemonnier P, Onaisi A, Gérard C, Koutsabeloulis N (2002) Geomechanics in reservoir simulation: Overview of coupling methods and field case study. Oil and Gas Science and Technology 57(5):471-483, DOI 10.2516/ogst:2002031

Menaceur H, Delage P, Tang AM, Conil N (2016) On the ThermoHydro-Mechanical Behaviour of a Sheared Callovo-Oxfordian Claystone Sample with Respect to the EDZ Behaviour. Rock Mechanics and Rock Engineering 49(5):1875-1888, DOI 10.1007/s00603-015-0897-5, URL "http://dx.doi.org/10.1007/s00603-015-0897-5

Mindlin R (1964) Micro-structure in linear elasticity. Archive for Rational Mechanics and Analysis 16(1), DOI 10.1007/BF00248490

Mühlhaus HB (1986) Scherfugenanalyse bei granularem Material im Rahmen der Cosserat-Theorie. Ingenieur-Archiv 56:389-399, DOI 10.1007/BF02570619

Mühlhaus HB, Vardoulakis I (1987) The thickness of shear bands in granular materials. Géotechnique 37(3):271-283, DOI 10.1680/geot.1987.37.3.271, URL

http://www.icevirtuallibrary.com/content/article/10.1680/geot.1987.37.3.271 
Needleman A (1988) Material rate dependence and mesh sensitivity in localization problems. Computer Methods in Applied Mechanics and Engineering 67(1):6985, DOI 10.1016/0045-7825(88)90069-2

Papanastasiou PC, Vardoulakis I (1992) Numerical treatment of progressive localization in relation to borehole stability. International Journal for Numerical and Analytical Methods in Geomechanics 16(6):389-424, DOI 10.1002/nag. 1610160602, URL http://doi.wiley.com/10.1002/nag.1610160602

Papanastasiou PC, Zervos A (2016) Numerical modelling of strain localization. In: ALERT Doctoral School 2016. Modelling of instabilities and bifurcation in Geomechanics, pp 133-164

Pijaudier CG, Bazant ZP (1987) Non local Damage Theory. Journal of engineering mechanics 113(10):1512-1533, DOI http: //dx.doi.org/10.1061/(ASCE)0733-9399(1987)113:10(1512), URL http://dx.doi.org/10.1061/(ASCE)0733-9399(1987)113:10(1512)

Poulet T, Paesold M, Veveakis M (2016) Multi-Physics Modelling of Fault Mechanics Using REDBACK: A Parallel Open-Source Simulator for Tightly Coupled Problems. Rock Mechanics and Rock Engineering pp 1-17, DOI 10.1007/s00603-016-0927-y

Rattez H, Stefanou I, Veveakis M, Sulem J, Poulet T (2017) Localisation of deformation for shearing of a rock layer with Cosserat microstructure and different couplings. In: 11th International Workshop on Bifurcation and Degradation in Geomaterials

Rattez H, Stefanou I, Sulem J (2018a) The importance of Thermo-HydroMechanical couplings and microstructure to strain localization in 3D continua with application to seismic faults. Part I: Theory and linear stability analysis. Journal of the Mechanics and Physics of Solids 115:54-76, DOI 10.1016/j.jmps.2018.03.004

Rattez H, Stefanou I, Sulem J, Veveakis M, Poulet T (2018b) The importance of Thermo-Hydro-Mechanical couplings and strain localisation in 3D continua with microstructure . Part II : Numerical implementation and post-bifurcation analysis. Journal of the Mechanics and Physics of Solids 115:1-29, DOI 10.1016/ j.jmps.2018.03.003

Regenauer-Lieb K, Poulet T, Veveakis M, Alevizos S (2017) The dynamics of multiscale, multiphysics faults: Part I - The long-term behaviour of the lithosphere. Tectonophysics (October), DOI 10.1016/j.tecto.2017.06.011

Rice JR (1975) On the Stability of Dilatant Hardening for Saturated Rock Masses. Journal of Geophysical Research 80(11):1531-1536, DOI 10.1029/JB080i011p01531, URL http://www .agu.org/pubs/crossref/1975/JB080i011p01531.shtml

575 Rice JR (2006a) Heating and weakening of faults during earthquake slip. Journal of Geophysical Research 111(B5), DOI 10.1029/2005JB004006, URL http://www .agu.org/pubs/crossref/2006/2005JB004006.shtml

Rice JR (2006b) Heating and weakening of faults during earthquake slip. Journal of Geophysical Research: Solid Earth 111(5), DOI 10.1029/2005JB004006

${ }_{580}$ Sammis CG, Ben-Zion Y (2008) Mechanics of grain-size reduction in fault zones. Journal of Geophysical Research: Solid Earth 113(2):1-12, DOI 10.1029/ 2006JB004892

Scuderi MM, Collettini C (2016) The role of fluid pressure in induced vs. triggered seismicity: Insights from rock deformation experiments on 
carbonates. Scientific Reports 6(April):1-9, DOI 10.1038/srep24852, URL http://dx.doi.org/10.1038/srep24852

Segall P, Rice JR (2006) Does shear heating of pore fluid contribute to earthquake nucleation? Journal of Geophysical Research: Solid Earth 111(9):1-17, DOI 10.1029/2005JB004129

Sibson RH (2003) Thickness of the seismic slip zone. Bulletin of the Seismological Society of America 93(3):1169-1178, DOI 10.1785/0120020061

Sleep NH (1999) Rate- and state-dependent friction of intact rock and gouge. Journal Of Geophysical Research 104(B8):17,847-17,855, DOI 10.1029/ 1999JB900185

Stefanou I, Sulem J (2014a) Chemically induced compaction bands: Triggering conditions and band thickness. Journal of Geophysical Research: Solid Earth 119(2):880-899, DOI 10.1002/2013JB010342, URL http://doi .wiley.com/10.1002/2013JB010342

Stefanou I, Sulem J (2014b) Chemically induced compaction bands: Triggering conditions and band thickness. Journal of Geophysical Research: Solid Earth 119(2):880-899, DOI 10.1002/2013JB010342, URL http://doi.wiley.com/10.1002/2013JB010342

Stefanou I, Sulem J, Rattez H (2017) Cosserat approach to localization in geomaterials. In: Handbook of Nonlocal Continuum Mechanics for Materials and

605 Structures, Springer

Sulem J, Famin V (2009) Thermal decomposition of carbonates in fault zones: Slipweakening and temperature-limiting effects. Journal of Geophysical Research 114(B3):1-14, DOI 10.1029/2008JB006004

Sulem J, Stefanou I, Veveakis M (2011) Stability analysis of undrained adiabatic shearing of a rock layer with Cosserat microstructure. Granular Matter 13(3):261-268, DOI 10.1007/s10035-010-0244-1

Tung R, Poulet T, Alevizos S, Veveakis M, Regenauer-Lieb K (2017) Shear heating in creeping faults changes the onset of convection. Geophysical Journal International 211(1):270-283

Unterreiner P (1994) Contribution à l'étude et à la modélisation numérique des sols cloués : application au calcul en déformation des ouvrages de soutènement. $\mathrm{PhD}$ thesis, Ecole Nationale des Ponts et Chaussées

Vardoulakis I (1985) Stability and bifurcation of undrained, plane rectilinear deformations on water-saturated granular soils. International Journal for Numerical and Analytical Methods in Geomechanics 9(5):399-414, DOI 10.1002/nag. 1610090502, URL http://doi.wiley.com/10.1002/nag. 1610090502

Vardoulakis I (1986) Dynamic stability analysis of undrained simple shear on water-saturated granular soils. International Journal for Numerical and Analytical Methods in Geomechanics 10(2):177-190, DOI 10.1002/nag.1610100206, URL http://doi.wiley.com/10.1002/nag.1610100206

Vardoulakis I, Sulem J (1995) Bifurcation Analysis in Geomechanics. Blackie, Glascow

Vardoulakis I, Unterreiner P (1995) Interfacial localisation in simple shear tests on a granular medium modelled as a Cosserat continuum. Studies in Applied Mechanics 42(C):487-512, DOI 10.1016/S0922-5382(06)80023-1

Vardoulakis I, Shah KR, Papanastasiou P (1992) Modelling of Tool-Rock Shear Interfaces Using Gradient-dependent Flow Theory of Plasticity. International Journal of Rock Mechanics and Mining Sciences \& Geomechanics Abstracts 
$29(6): 573-582$

${ }_{635}$ Wentworth CK (1922) A Scale of Grade and Class Terms for Clastic Sediments. The Journal of Geology 30(5):377-392

Wibberley CA, Shimamoto T (2003) Internal structure and permeability of major strike-slip fault zones: the Median Tectonic Line in Mie Prefecture, Southwest Japan. Journal of Structural Geology 25(1):59-78, DOI 10.1016/S0191-8141(02) 00014-7

Wibberley CAJ, Yielding G, Toro GD, Wibberley CAJ, Yielding G, Toro GDI, Einstein A, House NB, Lane NB, Pe L (2008) Recent advances in the understanding of fault zone internal structure : a review. Geological Society, London, Special Publication pp 5-33, DOI 10.1144/SP299.2

Zervos A, Papanastasiou P, Vardoulakis I (2001) A finite element displacement formulation for gradient elastoplasticity. International Journal for Numerical Methods in Engineering 50(6):1369-1388, DOI 10.1002/1097-0207(20010228)50: 6〈1369::AID-NME72 $>3.0 . \mathrm{CO} ; 2-\mathrm{K}$
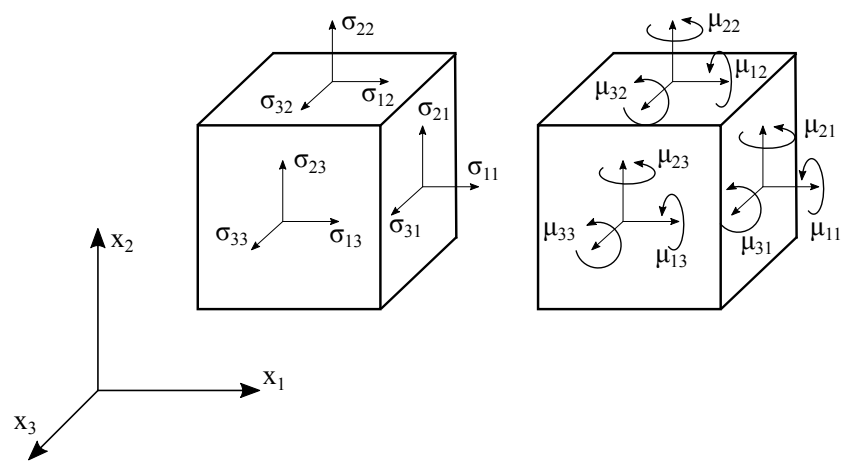

Fig. 1 Representation of stress and couple-stress components in a Cosserat continuum.

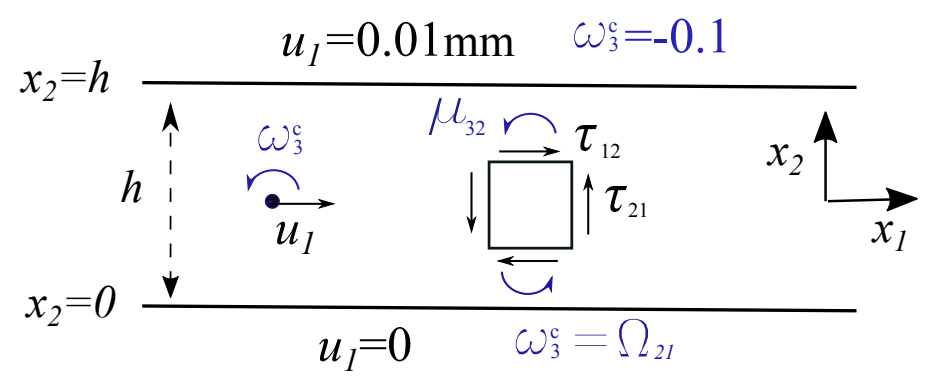

Fig. 2 Boundary conditions for the simple shear of a layer consisting of linear elastic Cosserat material (Boundary layer effect) 

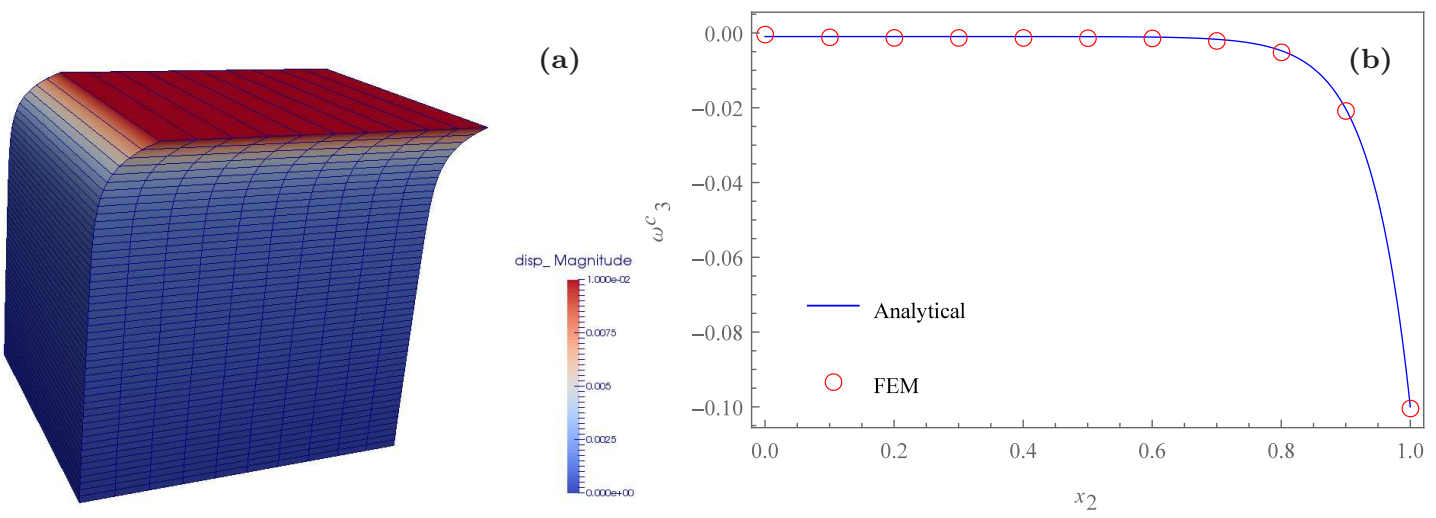

Fig. 3 (a) Results of the FEM simulation for the elasticity test with 50 elements in the vertical direction (Deformation Scale Factor: 20) (b) Comparison of the profiles of the Cosserat rotation $\omega_{3}^{c}$ obtained from the analytical solution and the FEM simulation with 50 points in the $x_{2}$ direction

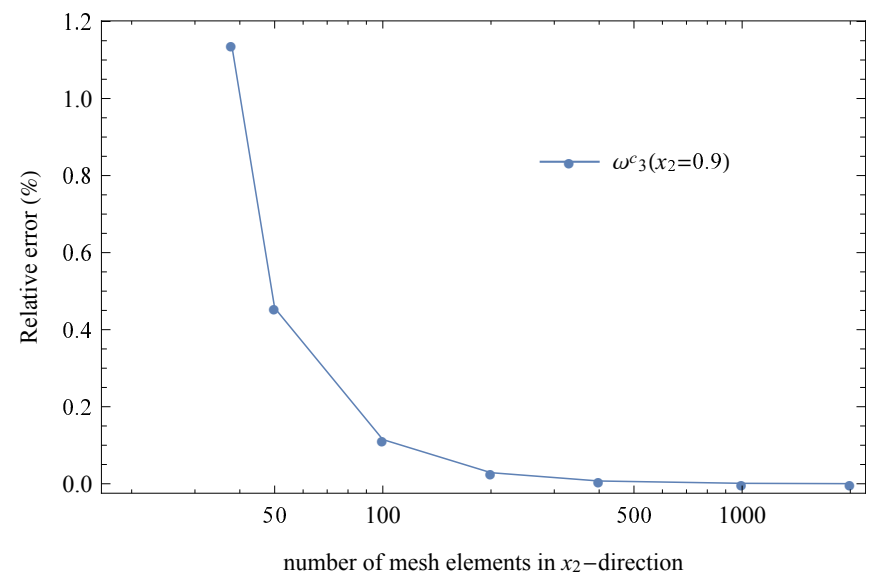

Fig. 4 Relative error of the results obtained by FEM for the Cosserat rotation $\omega_{3}^{c}\left(x_{2}=0,9\right)$ as a function of the number of elements in the $x_{2}$-axis direction

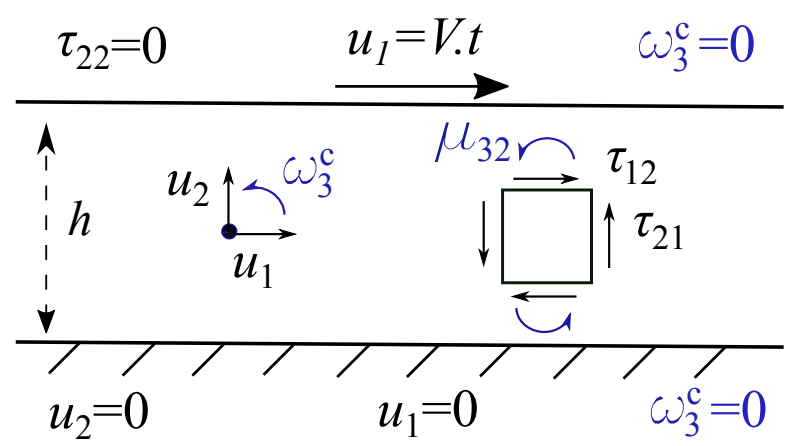

Fig. 5 Pure shear of an infinite layer with Cosserat microstructure. Notations and boundary conditions for the plasticity tests. 

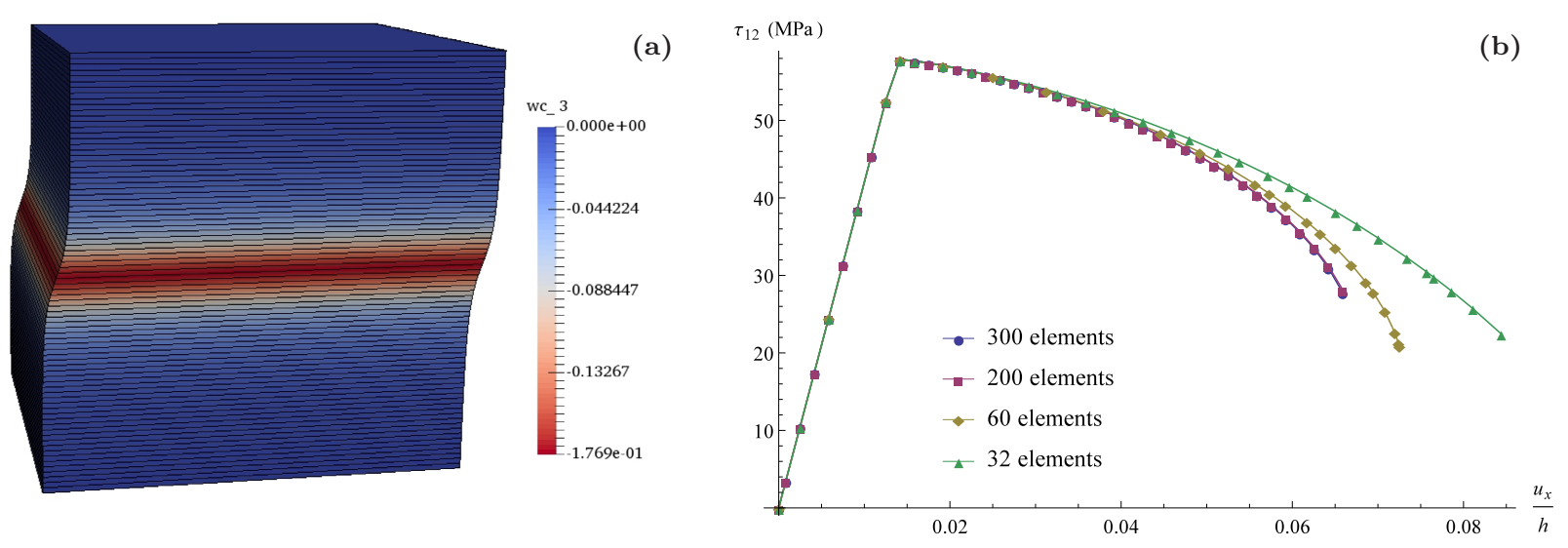

Fig. 6 Results of the FEM simulation for the example of the infinite layer under simple shear considering an elasto-plastic Cosserat continuum and von Mises yield criterion. (a)The Cosserat rotation is plotted on the deformed mesh with 80 elements in the vertical direction $\left(\tau_{12}=26 \mathrm{MPa}\right.$, Deformation Scale Factor: 1). (b)Stress-strain response of the layer plotted for different number of elements in the vertical direction.

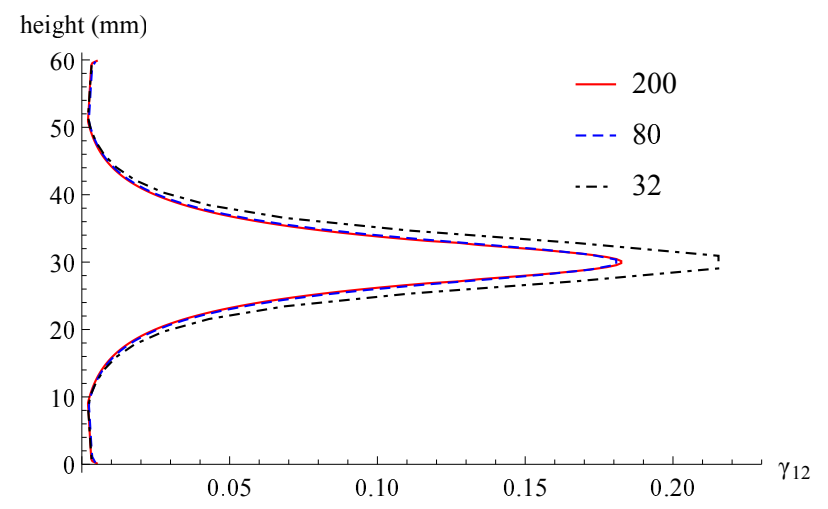

Fig. 7 Shear strain profile $\gamma_{12}$ from FEM simulations of an elasto-plastic infinite layer plotted for different number of elements in the vertical direction $\left(\tau_{12}=26 \mathrm{MPa}\right)$. 
(a)
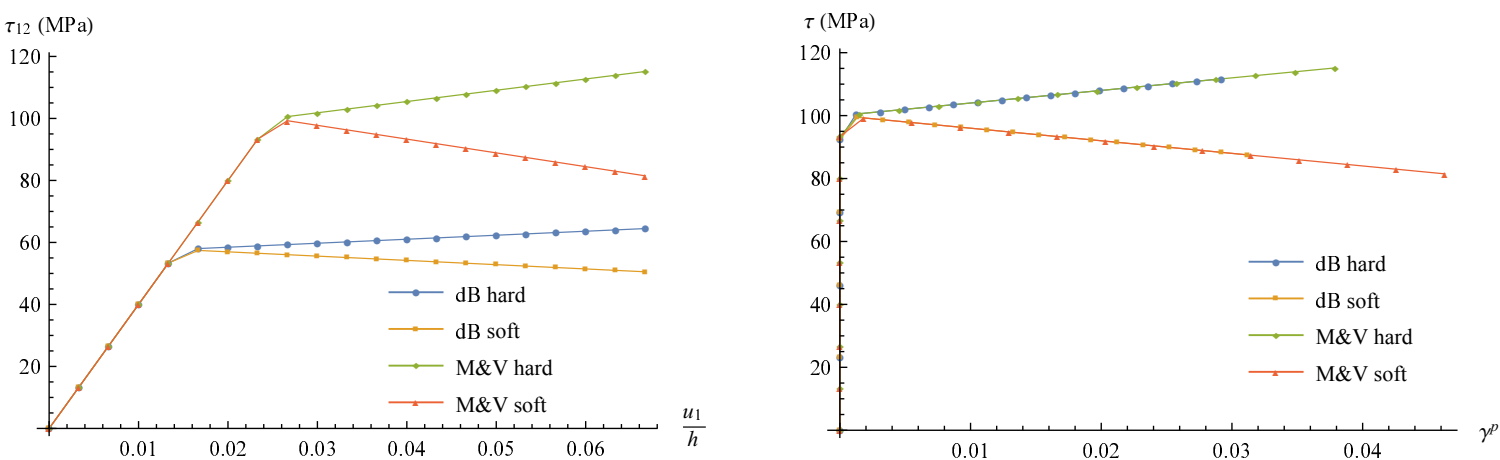

Fig. 8 FEM results for the shearing of one element: (a) Stress-strain response and (b) Shear stress invariant as a function of the plastic shear strain invariant, with coefficients of Mühlhaus and Vardoulakis (1987) ("M\&V") and de Borst (1991) ("dB").

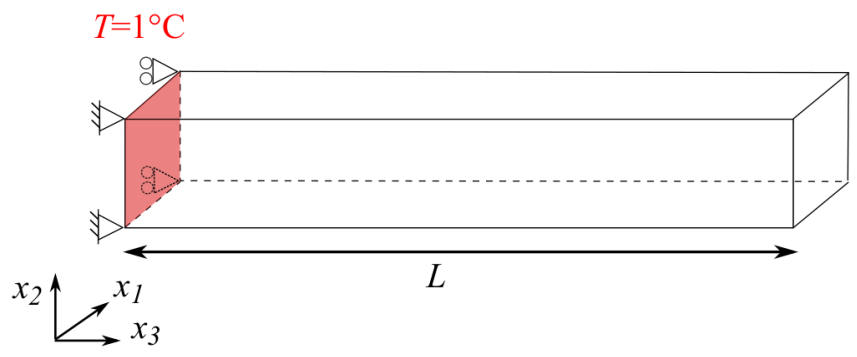

Fig. 9 Geometry and boundary conditions for the rod used in the first test for ThermoMechanical couplings.

(a)

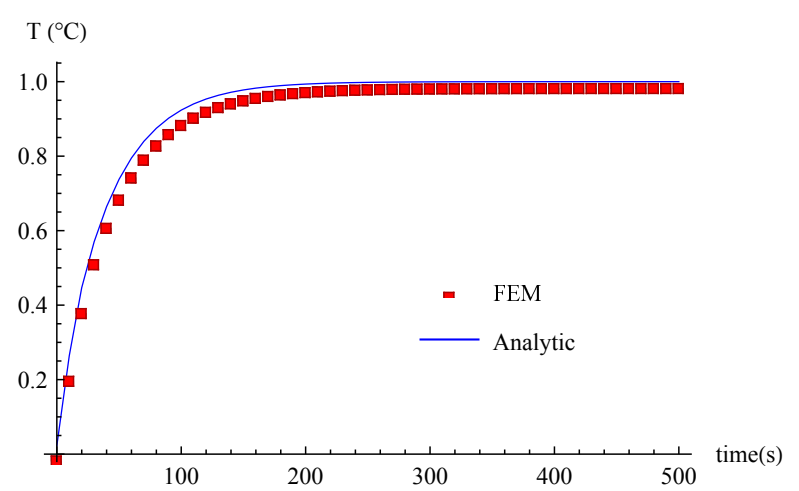

(b)

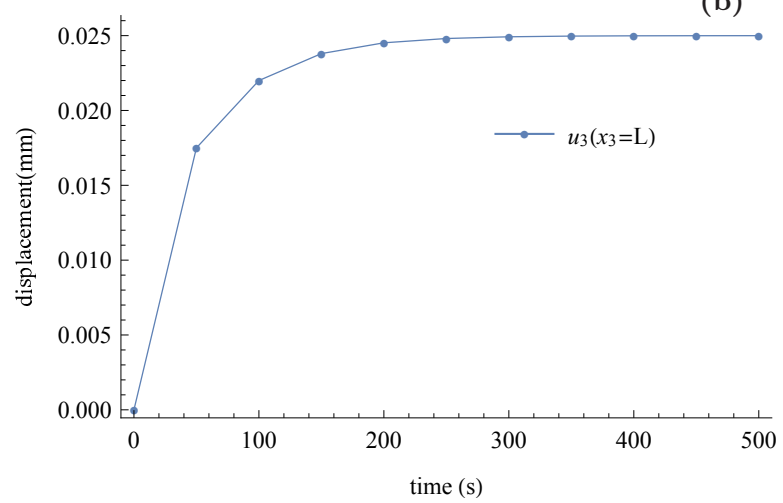

Fig. 10 Results of the FEM simulations for the heated $\operatorname{rod}\left(c_{t h}=1 \mathrm{~mm}^{2} / \mathrm{s}\right)$ : (a) Temperature evolution in the middle of the rod with time compared to the analytical solution, (b) Normal displacement at the end of the rod $u_{3}\left(x_{3}=L\right)$ due to thermal dilation. 


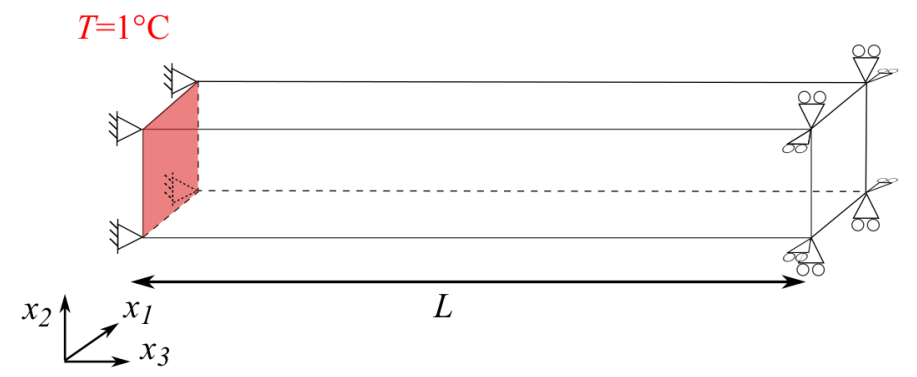

Fig. 11 Geometry and boundary conditions for the second rod used in the tests for ThermoMechanical couplings.

(a)

(b)
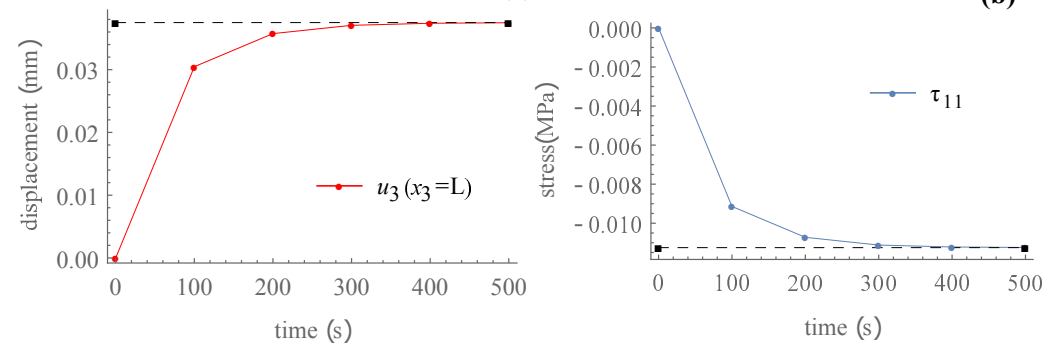

Fig. 12 Results for the heated elastic confined rod: (a) stress $\tau_{11}$ as a function of time, (b) displacement $u_{3}$ at the end of the rod as a function of time.

(a)

(b)
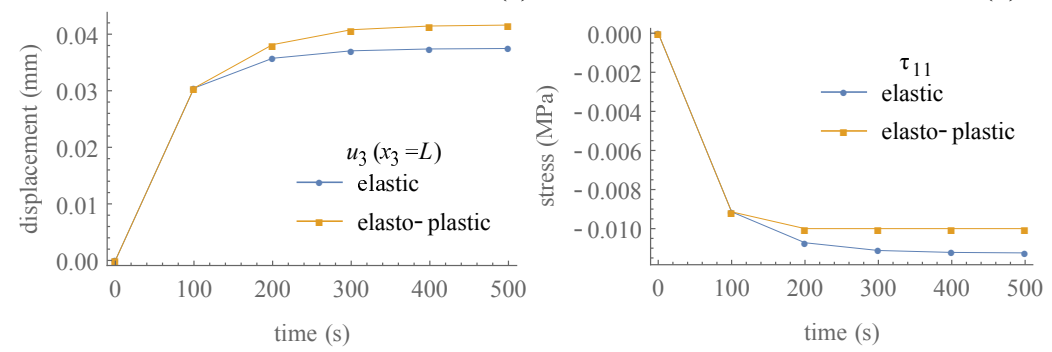

Fig. 13 Comparison of results for (a) the displacement $u_{3}\left(x_{3}=L\right)$ and (b) the stress $\tau_{11}$, for the heated confined rod in elasticity and plasticity. 


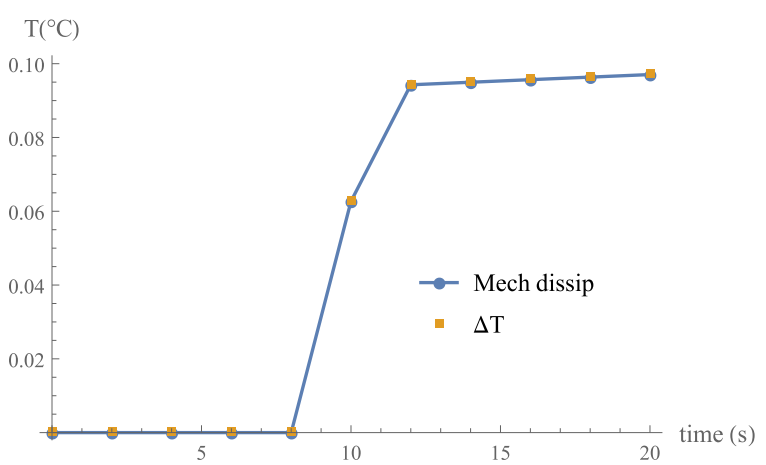

Fig. 14 Comparison of the temperature increment as a function of time for the shearing test with the mechanical dissipation term considered in the energy balance equation.

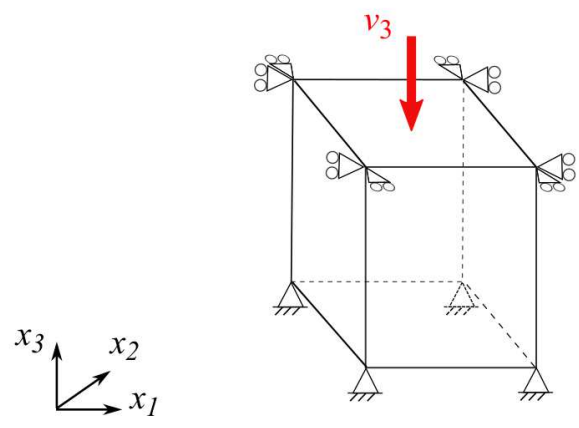

Fig. 15 Geometry and boundary conditions used for Hydro-Mechanical oedometer tests. 


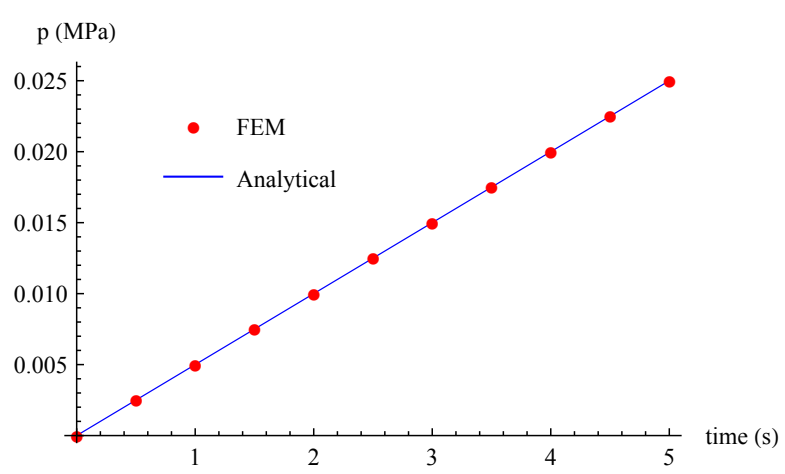

Fig. 16 Comparison of numerical and analytical results for the pore pressure in a undrained oedometric test $\left(\beta^{*}=2 \mathrm{MPa}^{-1}\right)$

(a)
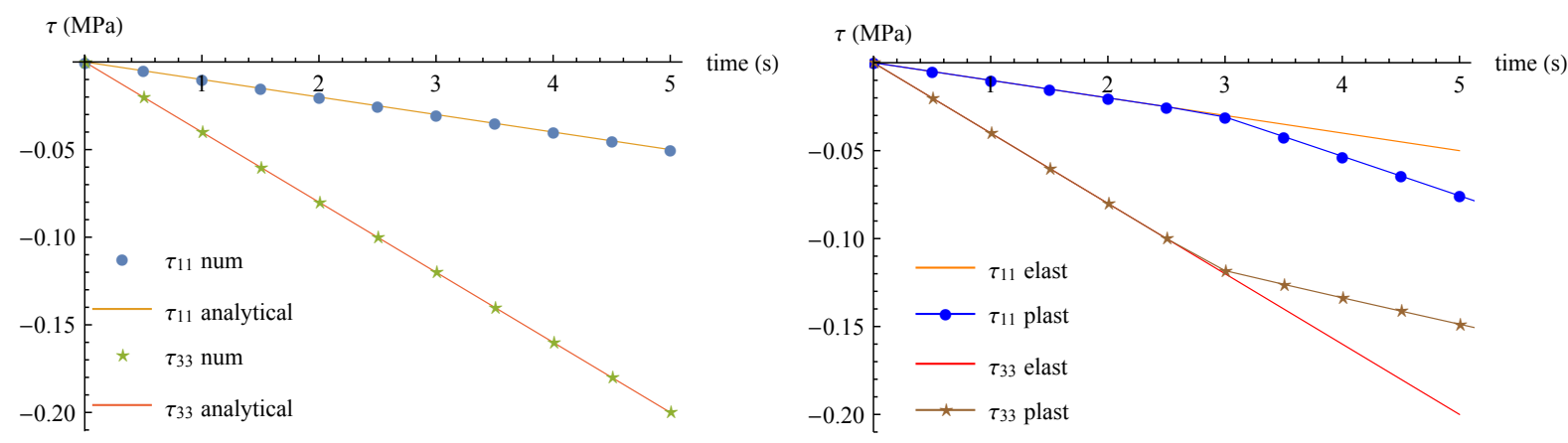

Fig. 17 Evolution of the stresses with time in a undrained oedometric test: (a)Comparison of FEM and analytical results for an elastic material (b)Comparison of elastic and elasto-plastic materials.

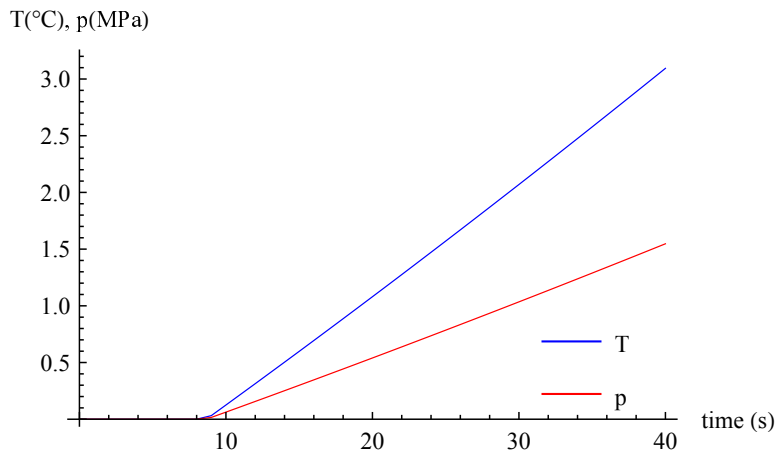

Fig. 18 Evolution of the pore pressure and temperature for the sheared layer of $60 \mathrm{~mm}$ with a mesh of only one element considering simplified THM couplings (no diffusion). 


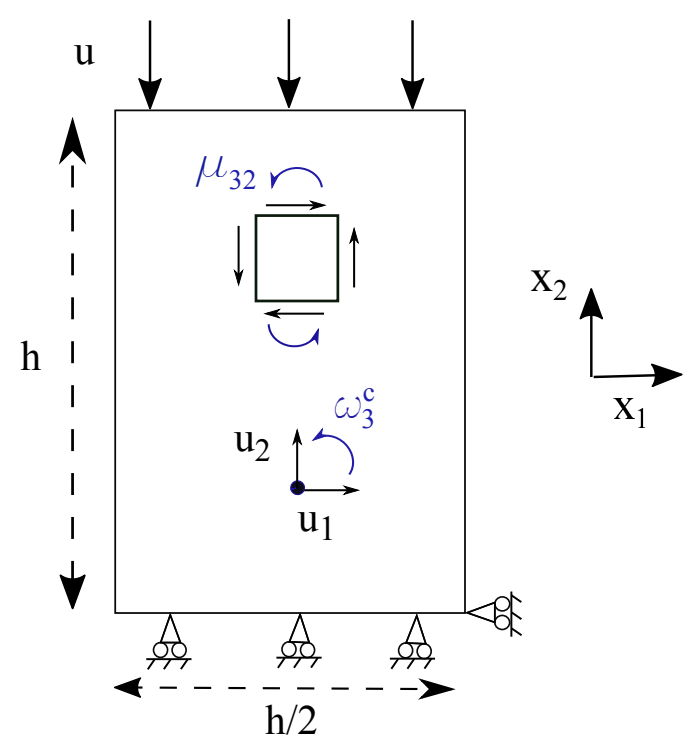

Fig. 19 Geometry and boundary conditions for the biaxial test

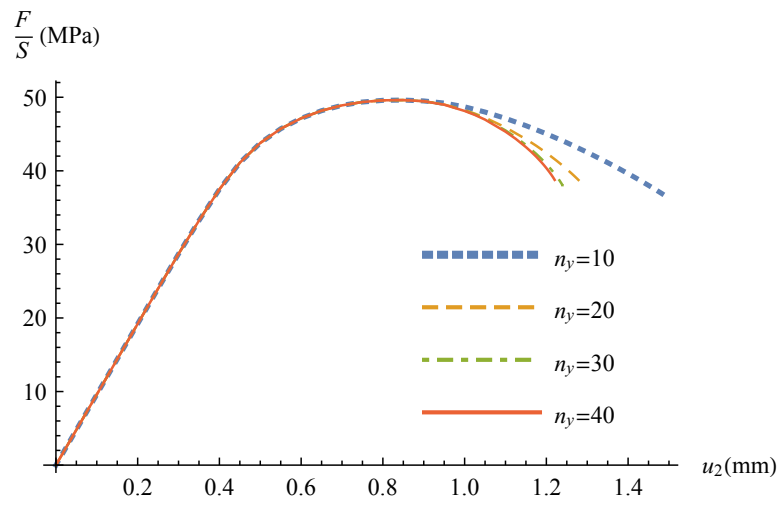

Fig. 20 Stress-strain responses of undrained biaxial tests on sandstones for different meshes identified by the number of elements in the vertical direction $n_{y}\left(R=2 \mathrm{~mm}, K_{1}=10\right)$. 


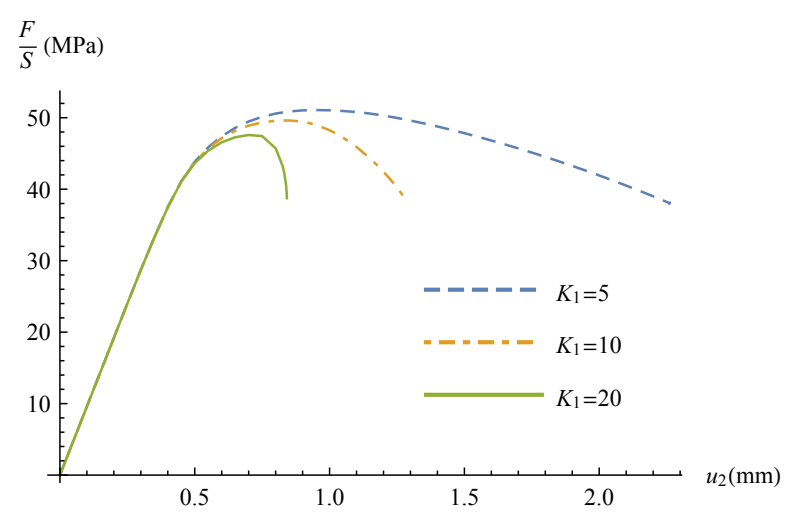

Fig. 21 Stress-strain responses of undrained biaxial tests on sandstones for different values of the parameter $H_{s}$ in the parabolic hardening evolution $\left(R=2 \mathrm{~mm}, n_{y}=30\right)$.

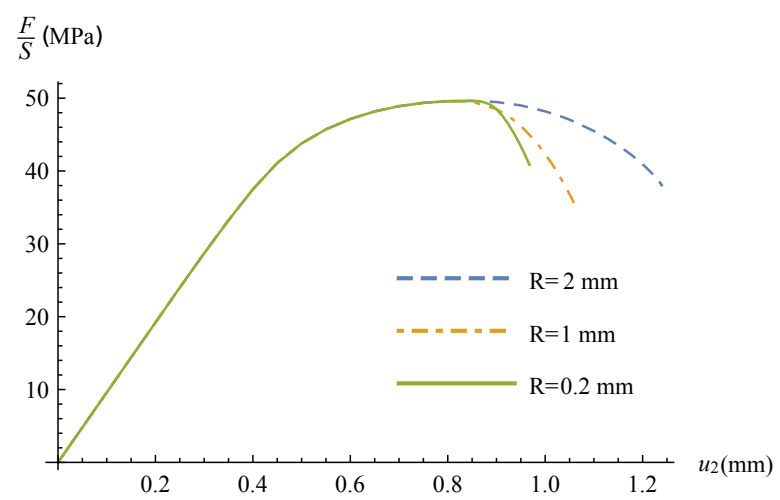

Fig. 22 Stress-strain responses of undrained biaxial tests on sandstones for different values of the characteristic size of the microstructure $R\left(K_{1}=10, n_{y}=30\right)$.

$\mathrm{R}=2 \mathrm{~mm}$

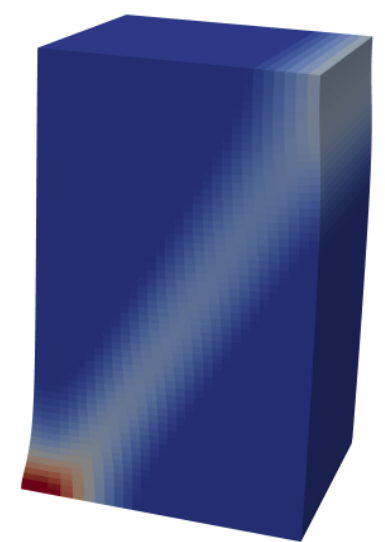

$\mathrm{R}=0.2 \mathrm{~mm}$

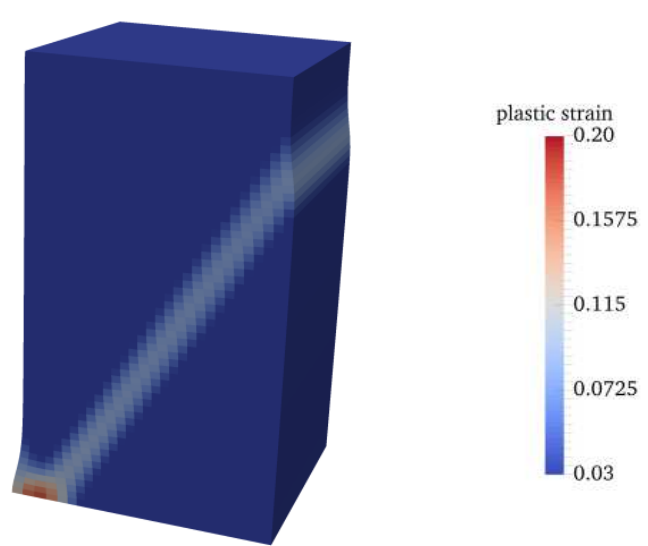

Fig. 23 Deformed mesh of biaxial tests on sandstones for different values of the characteristic size of the microstructure $R$. 


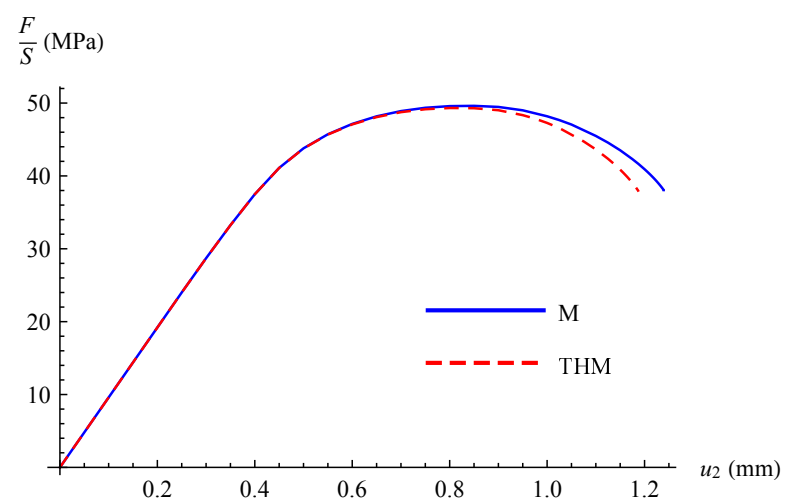

Fig. 24 Comparison of the stress-strain responses of biaxial tests for sandstones taking into account THM couplings or only the mechanical behavior $(\mathrm{M})\left(K_{1}=10, R=2 \mathrm{~mm}\right.$ and $\left.n_{y}=30\right)$.

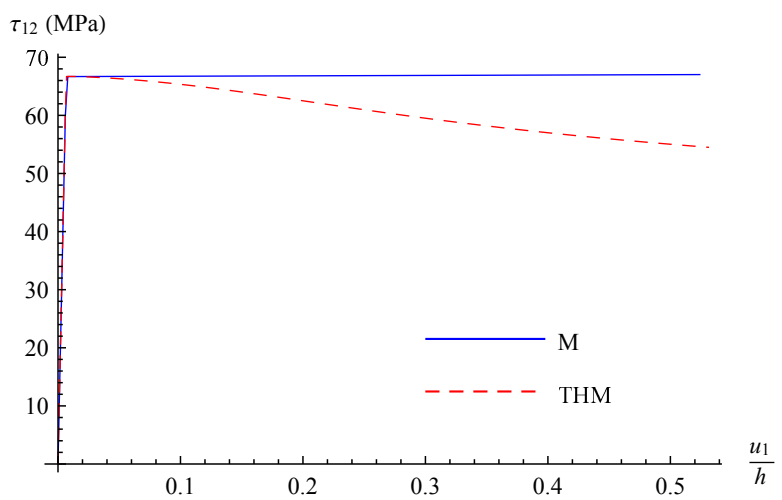

Fig. 25 Comparison of the stress-strain responses of fault zones modeled as infinite sheared layers taking into account THM couplings or only the mechanical behavior considering perfect plasticity and the parameters summarized in Table 2

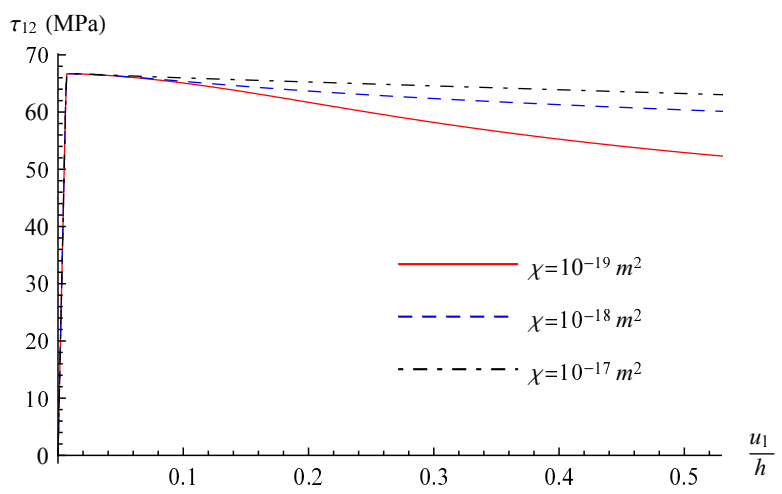

Fig. 26 Stress-strain response of a fault zone modeled as an infinite sheared layer taking into account THM couplings for different values of the permeability $\chi$. 


$$
\chi=10^{-17} \mathrm{~m}^{2}
$$

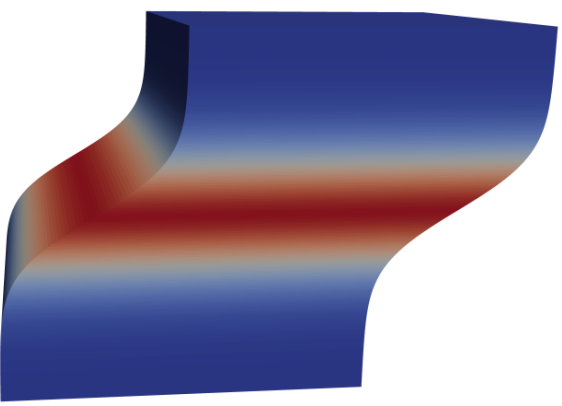

plastic strain

0.0 0.425

0.85

1.275

1.7

1.7

Fig. 27 FEM results of the plastic strain profile plotted on the deformed mesh for two different values of the permeability $\chi$. 